\title{
Checklist of the helminth parasites of the genus Profundulus Hubbs, 1924 (Cyprinodontiformes, Profundulidae), an endemic family of freshwater fishes in Middle-America
}

\author{
Carlos Daniel Pinacho-Pinacho',2, Martín García-Varela', Jesús S. Hernández-Orts', \\ Carlos A. Mendoza-Palmero', Ana L. Sereno-Uribe', Emilio Martínez-Ramírez ${ }^{3}$, \\ Leopoldo Andrade-Gómez', Alejandra López-Jiménez', Eduardo Hernández-Cruz', \\ Gerardo Pérez-Ponce de León'
}

I Departamento de Zoología, Instituto de Biología, Universidad Nacional Autónoma de México, Apartado Postal 70-153, C. P. 14510, México, D. F., México 2 Posgrado en Ciencias Biológicas, Instituto de Biología, Universidad Nacional Autónoma de México, Apartado Postal 70-153, C.P. 04510, México, D.F., México 3 Departamento de Investigación, Área de Acuacultura, Centro Interdisciplinario de Investigación para el Desarrollo Integral Regional, Unidad Oaxaca, Instituto Politécnico Nacional. Hornos Núm. 1003, Col. Noche Buena, Santa Cruz Xoxocotlán, 71230 Oaxaca, México

Corresponding author: Carlos Daniel Pinacho-Pinacho (danyboy_jd26@hotmail.com)

Academic editor: D. Gibson | Received 18 June 2015 | Accepted 27 August 2015 | Published 28 September 2015

http://zoobank.org/35E4923C-71F1-4F7F-9DA2-CAFAC7E0F6D5

Citation: Pinacho-Pinacho CD, García-Varela M, Hernández-Orts JS, Mendoza-Palmero CA, Sereno-Uribe AL, Martínez-Ramírez E, Andrade-Gómez L, López-Jiménez A, Hernández-Cruz E, de León GP-P (2015) Checklist of the helminth parasites of the genus Profundulus Hubbs, 1924 (Cyprinodontiformes, Profundulidae), an endemic family of freshwater fishes in Middle-America. ZooKeys 523: 1-30. doi: 10.3897/zookeys.523.6088

\begin{abstract}
From December 2012 to November 2014, 267 fish belonging to the family Profundulidae (representing nine of the 11 species of the genus Profundulus) were collected in 26 localities of Middle-America, across southern Mexico, Guatemala, and Honduras, comprising the distribution range of the genus, and analyzed for helminth parasites. Additionally, a database with all ten available published accounts of the helminth parasite fauna of this genus (the only genus within the family) was assembled. Based on both sources of information, a checklist containing all the records was compiled as a tool to address future questions in the areas of evolutionary biology, biogeography, ecology and phylogeography of this host-parasite association. The helminth parasite fauna of this fish group consists of 20 nominal species, classified in 17 genera and 14 families. It includes six species of adult digeneans, five metacercariae, two monogeneans, one adult cestode, three adult nematodes and three larval nematodes. The profundulid
\end{abstract}

Copyright Carlos Daniel Pinacho-Pinacho et al. This is an open access article distributed under the terms of the Creative Commons Attribution License (CC BY 4.0), which permits unrestricted use, distribution, and reproduction in any medium, provided the original author and source are credited. 
fishes are parasitized by a specialized group of helminth species (e.g. Paracreptotrema blancoi sensu Salgado-Maldonado et al. (2011b), Paracreptotrema profundulusi Salgado-Maldonado, Caspeta-Mandujano \& Martínez Ramírez, 2011, Phyllodistomum spinopapillatum Pérez-Ponce de León, Pinacho-Pinacho, Mendoza-Garfias \& García-Varela, 2015, Spinitectus humbertoi Mandujano-Caspeta \& Moravec, 2000, S. mariaisabelae Caspeta-Mandujano Cabañas-Carranza \& Salgado-Maldonado, 2007 and Rhabdochona salgadoi Mandujano-Caspeta \& Moravec, 2000), representing the core helminth fauna that are not shared with other Middle-American fish species.

\section{Keywords}

Killifish, Profundulidae, Middle-America, Digenea, Monogenea, Cestoda, Nematoda

\section{Introduction}

The information gathered regarding the composition of the helminth parasites of freshwater fishes of Mexico has increased in recent years (Pérez-Ponce de León and Choudhury 2010). The large number of published papers contributing to the inventory of the helminth parasite fauna of fish hosts in the last decades allowed Luque and Poulin (2007) to suggest that Mexico stands out as a hotspot of parasite diversity in freshwater fishes. The species composition of the helminth fauna of some freshwater fish families, such as the Cichlidae and Goodeidae, is well known (Vidal-Martínez et al. 2001; Martínez-Aquino et al. 2014).

The distribution of the Profundulidae extends along the Atlantic and Pacific Ocean slopes of southern Mexico, Guatemala, El Salvador and Honduras (Miller 1955; Miller et al. 2005; Doadrio et al. 1999; Matamoros and Schaeffer 2010; Matamoros et al. 2012); from the Río Aguacatillo (a tributary of the Laguna Tres Palos) in Guerrero, Mexico to the Río Nacaome in Honduras, and on the Atlantic slope from the Río Quiotepec (the Río Papaloapan drainage basin) in Oaxaca, to the Río Ulúa, Honduras (MartínezRamírez et al. 2004; Matamoros et al. 2012). The family contains a single genus (Profundulus Hubbs, 1924), the current species composition of which is a matter of debate, since some authors recognize only eight valid species (Matamoros and Schaeffer 2010; Matamoros et al. 2012), whereas others (see Doadrio et al. 1999: Martínez-Ramírez et al. 2004) also recognize $P$. balsanus Ahl, 1935 as a valid species, as well as two undescribed taxa, Profundulus sp. 1, and Profundulus sp. 2, which are currently being described by one of us (EMR). In addition to this, a molecular analysis of nuclear and mitochondrial genes, which will be published elsewhere, corroborates the validity of these three species (Ornelas-García, pers. comm.). Irrespective of the species composition, all Profundulus species represent an endemic lineage in Middle-America that has probably inhabited this region since the Pliocene and perhaps even the Miocene (Miller 1955; Doadrio et al. 1999; González-Diaz et al. 2005; Matamoros and Schaeffer 2010).

Records of the helminth parasite fauna of this family began with Caspeta-Mandujano and Moravec (2000), who described two nematode species, Spinitectus humbertoi Mandujano-Caspeta \& Moravec, 2000 and Rhabdochona salgadoi Caspeta-Mandujano \& Moravec, 2000, from the intestine of Profundulus labialis (Günther, 1866) in 
Inzcuinatoyac, Guerrero, Mexico. To date, ten studies have been published regarding some aspects of the helminth parasite fauna of profundulids, including descriptions of new species, inventories in particular localities and analyses of the parasite community structure of particular host species (Caspeta-Mandujano et al. 2007; Velázquez-Velázquez et al. 2011; Salgado-Maldonado et al. 2011a, b; Pinacho-Pinacho et al. 2014; Salgado-Maldonado et al. 2014, 2015; Velazquez-Velazquez et al. 2015; Pérez-Ponce de León et al. 2015).

As a continuation of effort to provide a more complete inventory of the helminth parasite fauna of freshwater fishes in this geographical region, intensive samplings were conducted during the last few years of these killifishes throughout their ranges of distribution in an attempt to obtain empirical and robust data to enable further studies of this host-parasite association from a phylogenetic, phylogeographical and biogeographical perspective, with the aim of understanding the mechanisms that have shaped the evolutionary and biogeographical history of these fishes and their parasites in Middle-America. The main objectives of this research were (1) to compile all the available published accounts on the helminth fauna of profundulid fishes, and (2) to incorporate new data derived from our own survey work of the last few years.

\section{Material and methods}

Data compilation. A dataset of all published records of the helminth species reported from members of the family Profundulidae in Middle-America was compiled. The keywords "Parasit(e)*AND Profundulus" were used to conduct searches through the ISI Web of Science. All those studies whose datasets provided taxonomic information on the helminth taxa found in a sample of individual hosts were considered.

Current research. Original data from our own studies of the last two years were included. From December 2012 through to November 2014, 267 individual fish belonging to nine species of Profundulus, i.e. P. balsanus, P. candalarius Hubbs, 1924; P. guatemalensis (Günther, 1866); P. hildebrandi Miller, 1950; P. kreiseri Matamoros, Schaefer, Hernández \& Chakrabarty, 2012; P. labialis; P. portillorum Matamoros \& Schaefer, 2010; P. punctatus (Günther, 1866); and Profundulus sp. 2 (sensu Doadrio et al. 1999), were examined for helminth parasites. Fish were collected with seine nets and electrofishing in 26 localities of southern Mexico, Guatemala and Honduras (Fig. 1; Table 1). Fish were kept alive and examined for helminths no more than $4 \mathrm{~h}$ after their capture. Fish were killed with an overdose of anesthetic and placed in Petri dishes, and immediately examined for helminths. All the external surfaces, viscera and musculature of each host were examined under a stereomicroscope, and the helminths found were counted in situ. Adult digeneans and metacercariae, monogeneans, cestodes and nematodes were fixed in hot $4 \%$ neutral formalin, and additional specimens were fixed in $100 \%$ ethanol for future molecular studies. Some monogeneans were mounted in a mixture of glycerine-ammonium picrate (Ergens 1969) and in Gray-Wess medium (Vidal-Martínez et al. 2001) to study their sclerotized structures. Digeneans, cestodes and monogeneans used for morphological studies 


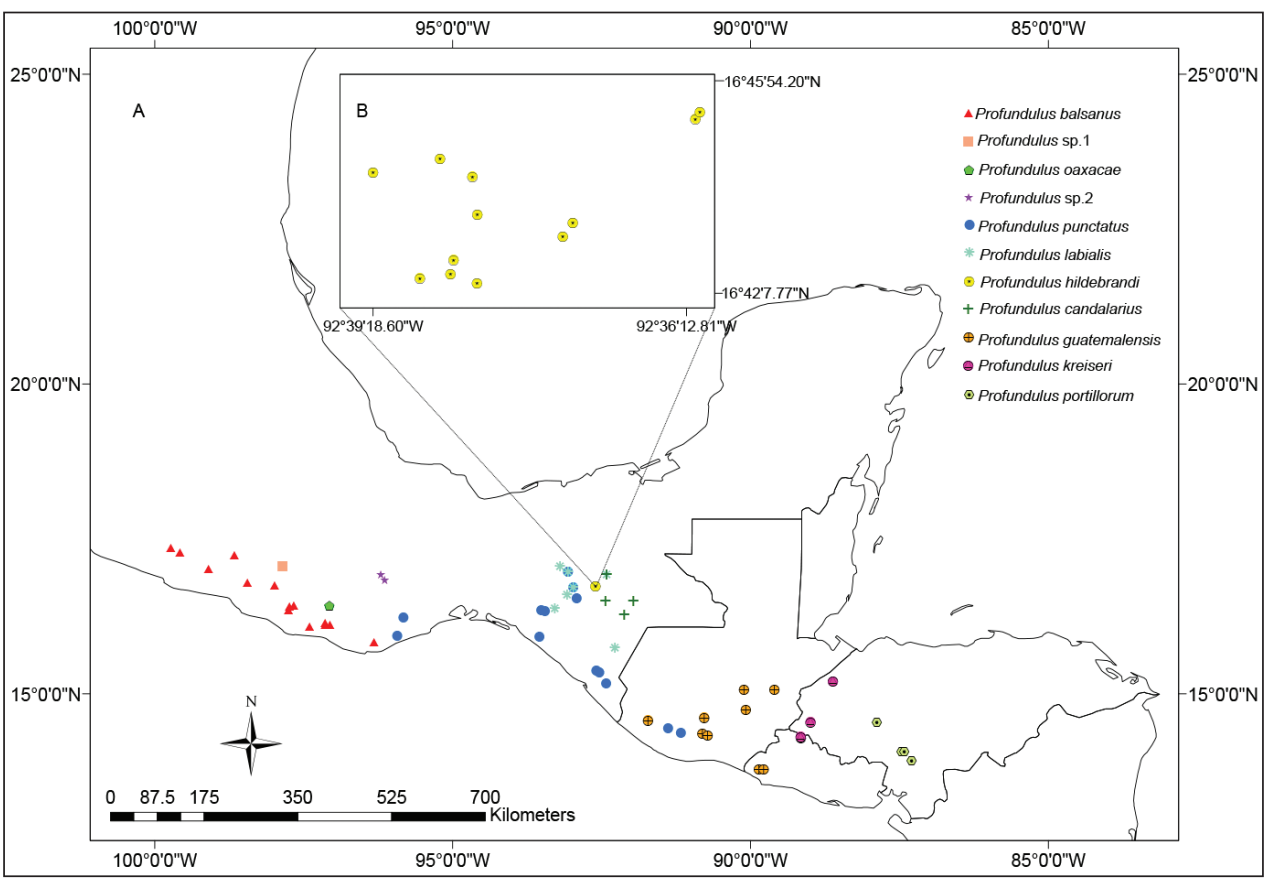

Figure I. A Map of Middle-America indicating the localities where specimens of Profundulidae have been have been examined for helminth parasites. Colors and symbols correspond for each species of Profundulus B Zoom of an endorrheic basin in San Cristóbal de la Casas, Chiapas, Mexico showing localities where the endemic fish $P$. hildebrandi was collected.

were stained with Mayer's paracarmine or iron acetocarmine, dehydrated using a graded alcohol series, cleared in methyl salicylate and mounted as permanent slides in Canada balsam. Nematodes were cleared with glycerine for light microscopy and stored in 70\% ethanol. Voucher specimens of some helminth species were deposited in the Colección Nacional de Helmintos (CNHE), Instituto de Biología, Universidad Nacional Autónoma de México, Mexico City, Mexico. Additionally, vouchers of Bothriocephalus acheilognathi Yamaguti, 1934 were deposited in the Helminthological Collection of the Institute of Parasitology (IPCAS), Biology Centre ASCR, České Budějovice, Czech Republic (accession numbers IPCAS C-15). Parameters of infection such as prevalence (\% infected) and mean intensity of infection (the average number of a particular species of parasite among the infected members of a particular host species) were calculated following Bush et al. (1997).

\section{Results}

The data analysis of both the bibliographic information and the survey work shows that 11 species of Profundulus (including undescribed species) studied for helminths, 
Table I. Localities in Mexico, Guatemala, El Salvador and Honduras where at least one helminth species has been recorded as a parasite of Profundulus. Localities marked with an asterisk $\left(^{*}\right)$ were sampled in this study. Collection sites (CS), locality (four letters code), geographical coordinates, country sampled and references are included. The collection site (CS) and locality code correspond with the localities referred in Table 2.

\begin{tabular}{|c|c|c|c|c|c|}
\hline \multirow{2}{*}{$\mathrm{CS}$} & \multirow{2}{*}{ Locality (code) } & \multicolumn{2}{|c|}{ Geographical coordinates } & \multirow{2}{*}{ Country } & \multirow{2}{*}{ References } \\
\hline & & $\mathbf{N}$ & W & & \\
\hline$(1)$ & Arroyo Inzcuinatoyac (Inzc) & $17^{\circ} 21^{\prime} 39^{\prime \prime}$ & $99^{\circ} 44^{\prime} 00^{\prime \prime}$ & México & $\begin{array}{l}\text { Caspeta-Mandujano and Moravec } \\
(2000)\end{array}$ \\
\hline$(2)$ & Río Suchiapa (Such) & $16^{\circ} 20^{\prime} 06^{\prime \prime}$ & $93^{\circ} 27^{\prime} 19^{\prime \prime}$ & México & Caspeta-Mandujano et al. (2007) \\
\hline$(3)$ & Ecosur (Ecos) & $16^{\circ} 42^{\prime} 55^{\prime \prime}$ & $92^{\circ} 37^{\prime} 28^{\prime \prime}$ & México & Velázquez-Velázquez et al. (2011) \\
\hline$(4)$ & La Albarrada (Alba) & $16^{\circ} 42^{\prime} 37^{\prime \prime}$ & $92^{\circ} 37^{\prime} 32^{\prime \prime}$ & México & Velázquez-Velázquez et al. (2011) \\
\hline$(5)$ & 5 de Marzo (5mar) & $16^{\circ} 42^{\prime} 34^{\prime \prime}$ & $92^{\circ} 38^{\prime} 14^{\prime \prime}$ & México & Velázquez-Velázquez et al. (2011) \\
\hline$(6)$ & El Puente (Puen) & $16^{\circ} 43^{\prime} 59^{\prime \prime}$ & $92^{\circ} 36^{\prime} 54^{\prime \prime}$ & México & Velázquez-Velázquez et al. (2011) \\
\hline$(7)$ & Arroyo Chamula (Cham) & $16^{\circ} 44^{\prime} 52^{\prime \prime}$ & $92^{\circ} 39^{\prime} 22^{\prime \prime}$ & México & Velázquez-Velázquez et al. (2011) \\
\hline$(8)$ & Peje de Oro (Peor) & $16^{\circ} 44^{\prime} 48^{\prime \prime}$ & $92^{\circ} 37^{\prime} 00^{\prime \prime}$ & México & Velázquez-Velázquez et al. (2011) \\
\hline$(9)$ & El Arcotete (Arco) & $16^{\circ} 45^{\prime} 57^{\prime \prime}$ & $92^{\circ} 31^{\prime} 43^{\prime \prime}$ & México & Velázquez-Velázquez et al. (2011) \\
\hline$(10)$ & Arenal (Aren) & $16^{\circ} 43^{\prime} 31^{\prime \prime}$ & $92^{\circ} 34^{\prime} 53^{\prime \prime}$ & México & Velázquez-Velázquez et al. (2011) \\
\hline$(11)$ & Agua de Pajarito (Paja) & $16^{\circ} 43^{\prime} 43^{\prime \prime}$ & $92^{\circ} 34^{\prime} 44^{\prime \prime}$ & México & Velázquez-Velázquez et al. (2011) \\
\hline$(12)$ & Laguna Soyul (Lsoy) & $16^{\circ} 46^{\prime} 01^{\prime \prime}$ & $92^{\circ} 31^{\prime} 39^{\prime \prime}$ & México & Velázquez-Velázquez et al. (2011) \\
\hline (13) & Río Ocotlán (Ocot) & -- & - & México & Salgado-Maldonado et al. (2011a) \\
\hline$(14)$ & Río Chicomosuelo (Chic) & $15^{\circ} 44^{\prime} 38^{\prime \prime}$ & $92^{\circ} 16^{\prime} 50^{\prime \prime}$ & México & Salgado-Maldonado et al. (2011a) \\
\hline$(15)$ & $\begin{array}{c}\text { Río Suchiapa, José María Garza } \\
\text { (Rsuc) }\end{array}$ & $16^{\circ} 36^{\prime} 36^{\prime \prime}$ & $93^{\circ} 05^{\prime} 03^{\prime \prime}$ & México & Salgado-Maldonado et al. (2011a) \\
\hline (16) & $\begin{array}{c}\text { Río San Juan, puente El Tablón, } \\
\text { Villa Flores (Saju) }\end{array}$ & $16^{\circ} 21^{\prime} 01^{\prime \prime}$ & $93^{\circ} 30^{\prime} 56^{\prime \prime}$ & México & Salgado-Maldonado et al. (2011a) \\
\hline (17) & Piedra Labrada (Labr) & $18^{\circ} 58^{\prime} 54^{\prime \prime}$ & $99^{\circ} 14^{\prime} 12^{\prime \prime}$ & México & Salgado-Maldonado et al. (2011b) \\
\hline (18) & Río La Soledad Carrizo (Carr) & $16^{\circ} 25^{\prime} 0.4^{\prime \prime}$ & $97^{\circ} 40^{\prime} 12.9^{\prime \prime}$ & México & $\begin{array}{l}\text { Salgado-Maldonado et al. (2011b) } \\
\text { Pinacho-Pinacho et al. (2014) }\end{array}$ \\
\hline (19) & Río San José de las Flores (Flor) & $16^{\circ} 24^{\prime} 21.5^{\prime \prime}$ & $97^{\circ} 44^{\prime} 22.6^{\prime \prime}$ & México & $\begin{array}{l}\text { Salgado-Maldonado et al. (2011b) } \\
\text { Pinacho-Pinacho et al. (2014) }\end{array}$ \\
\hline$(20)$ & $\begin{array}{l}\text { Río Santa Cruz Flores Magón } \\
\text { (Fmag)* }\end{array}$ & $16^{\circ} 21^{\prime} 6.1^{\prime \prime}$ & $97^{\circ} 45^{\prime} 38.3^{\prime \prime}$ & México & $\begin{array}{l}\text { Salgado-Maldonado et al. (2011b) } \\
\text { Pinacho-Pinacho et al. (2014) } \\
\text { Pérez-Ponce de León et al. (2015) } \\
\text { This study }\end{array}$ \\
\hline$(21)$ & Río Pichuaca (Pich)* & $16^{\circ} 05^{\prime} 34.2^{\prime \prime}$ & $97^{\circ} 24^{\prime} 18.1^{\prime \prime}$ & México & $\begin{array}{c}\text { Salgado-Maldonado et al. (2011b) } \\
\text { Pinacho-Pinacho et al. (2014) } \\
\text { This study }\end{array}$ \\
\hline$(22)$ & Río La Reforma (Refo) & $16^{\circ} 08^{\prime} 33.5^{\prime \prime}$ & $97^{\circ} 08^{\prime} 41.6^{\prime \prime}$ & México & $\begin{array}{l}\text { Salgado-Maldonado et al. (2011b) } \\
\text { Pinacho-Pinacho et al. (2014) }\end{array}$ \\
\hline (23) & Río Pueblo Viejo (Viej) ${ }^{*}$ & $16^{\circ} 06^{\prime} 22.3^{\prime \prime}$ & $97^{\circ} 03^{\prime} 47.8^{\prime \prime}$ & México & $\begin{array}{l}\text { Salgado-Maldonado et al. (2011b) } \\
\text { Pinacho-Pinacho et al. (2014) } \\
\text { Pérez-Ponce de León et al. (2015) } \\
\text { This study }\end{array}$ \\
\hline$(24)$ & $\begin{array}{c}\text { Río Santa María Huatulco } \\
\text { (Huat)* }\end{array}$ & $15^{\circ} 50^{\prime} 14.2^{\prime \prime}$ & $96^{\circ} 19^{\prime} 30.8^{\prime \prime}$ & México & $\begin{array}{l}\text { Salgado-Maldonado et al. (2011b) } \\
\text { Pinacho-Pinacho et al. (2014) } \\
\text { This study }\end{array}$ \\
\hline$(25)$ & Río Macuta (Macu) & - & - & México & Salgado-Maldonado et al. (2011b) \\
\hline (26) & $\begin{array}{l}\text { Río Templo, San Juan del Río } \\
\qquad(\text { Sjri })^{*}\end{array}$ & $16^{\circ} 53^{\prime} 56.3^{\prime \prime}$ & $96^{\circ} 09^{\prime} 57.3^{\prime \prime}$ & México & $\begin{array}{l}\text { Salgado-Maldonado et al. (2011b) } \\
\text { Pérez-Ponce de León et al. (2015) } \\
\text { This study }\end{array}$ \\
\hline$(27)$ & Arroyo Ojo de Agua (Ojag) & $16^{\circ} 13^{\prime} 38.6^{\prime \prime}$ & $95^{\circ} 49^{\prime} 36.6^{\prime \prime}$ & México & Salgado-Maldonado et al. (2011b) \\
\hline
\end{tabular}




\begin{tabular}{|c|c|c|c|c|c|}
\hline \multirow{2}{*}{ CS } & \multirow{2}{*}{ Locality (code) } & \multicolumn{2}{|c|}{ Geographical coordinates } & \multirow{2}{*}{ Country } & \multirow{2}{*}{ References } \\
\hline & & $\mathrm{N}$ & W & & \\
\hline (28) & Río La Laca (Rlac) & $17^{\circ} 14^{\prime} 09.3^{\prime \prime}$ & $98^{\circ} 39^{\prime} 55.7^{\prime \prime}$ & México & Salgado-Maldonado et al. (2014) \\
\hline (29) & Río Cahoapan (Caho) & $17^{\circ} 16^{\prime} 37.8^{\prime \prime}$ & $99^{\circ} 35^{\prime} 04.7^{\prime \prime}$ & México & Salgado-Maldonado et al. (2014) \\
\hline (30) & Río Tamarindo (Tama) & $17^{\circ} 00^{\prime} 36.5^{\prime \prime}$ & $99^{\circ} 06^{\prime} 0.8^{\prime \prime}$ & México & Salgado-Maldonado et al. (2014) \\
\hline (31) & Río del Aguacate (Agua) & $16^{\circ} 07^{\prime} 19^{\prime \prime}$ & $97^{\circ} 8^{\prime} 22.8^{\prime \prime}$ & México & Salgado-Maldonado et al. (2014) \\
\hline (32) & Arroyo los Sabinos (Sabi) & $16^{\circ} 25^{\prime} 39.9^{\prime \prime}$ & $97^{\circ} 4 ' 28.9^{\prime \prime}$ & México & Salgado-Maldonado et al. (2014) \\
\hline (33) & Río Chacalapa (Chac) & $15^{\circ} 55^{\prime} 54.8^{\prime \prime}$ & $95^{\circ} 56^{\prime} 00.3^{\prime \prime}$ & México & Salgado-Maldonado et al. (2014) \\
\hline (34) & Río Chicaguaxtla (Chic) & $17^{\circ} 03^{\prime} 30.30^{\prime \prime}$ & $97^{\circ} 51^{\prime} 32.52^{\prime \prime}$ & México & Salgado-Maldonado et al. (2014) \\
\hline (35) & Río Chico (Rchi) & $16^{\circ} 55^{\prime} 34.50^{\prime \prime}$ & $96^{\circ} 12^{\prime} 27.42^{\prime \prime}$ & México & Salgado-Maldonado et al. (2014) \\
\hline (36) & Amatenango del Río (Amri) & $16^{\circ} 31^{\prime} 22.2^{\prime \prime}$ & $92^{\circ} 25^{\prime} 10.7^{\prime \prime}$ & México & Salgado-Maldonado et al. (2014) \\
\hline (37) & Río Grande de Comitán (Rcom) & $16^{\circ} 16^{\prime} 49.9^{\prime \prime}$ & $92^{\circ} 07^{\prime} 21.1 "$ & México & Salgado-Maldonado et al. (2014) \\
\hline (38) & Arroyo ECOSUR (Aeco) & $16^{\circ} 42^{\prime} 27.3^{\prime \prime}$ & $92^{\circ} 36^{\prime} 54.8^{\prime \prime}$ & México & Salgado-Maldonado et al. (2014) \\
\hline (39) & Arroy & $16^{\circ} 33^{\prime} 13.7^{\prime \prime}$ & $92^{\circ} 28^{\prime} 34.9^{\prime \prime}$ & M & $\begin{array}{l}\text { Salgado-Maldonado et al. (2014) } \\
\text { Velazquez-Velazquez et al. (2015) }\end{array}$ \\
\hline$(40)$ & Río Rancho San Antonio (Rsan) & $16^{\circ} 58^{\prime} 30.9^{\prime \prime}$ & $93^{\circ} 03^{\prime} 44.7^{\prime \prime}$ & México & Salgado-Maldonado et al. (2014) \\
\hline (41) & Arroyo Tres Picos (Atpi) & $17^{\circ} 03^{\prime} 28.3^{\prime \prime}$ & $93^{\circ} 11^{\prime} 50.7^{\prime \prime}$ & México & Salgado-Maldonado et al. (2014) \\
\hline (42) & Río Nandalumi (Rnan) & $16^{\circ} 43^{\prime} 18.4^{\prime \prime}$ & $92^{\circ} 58^{\prime} 52.4^{\prime \prime}$ & México & Salgado-Maldonado et al. (2014) \\
\hline (43) & $\begin{array}{c}\text { Arroyo Ojo de Agua, El Canelar } \\
\text { (Cane) }\end{array}$ & $16^{\circ} 32$ & $92^{\circ}$ & $\mathrm{N}$ & Salgado-Mal \\
\hline$(44)$ & Río Nil (Rnil) & $14^{\circ} 33^{\prime} 54.4^{\prime \prime}$ & $91^{\circ} 43^{\prime} 25.4^{\prime \prime}$ & Guatemala & Salgado-Maldonado et \\
\hline (45) & Río el Cantil (Cant) & $14^{\circ} 21^{\prime} 22.6^{\prime \prime}$ & $90^{\circ} 48^{\prime} 30.4^{\prime \prime}$ & Guatemala & Salgado-Maldonado \\
\hline$(46)$ & Arroyo El Platanar (Apla) & $14^{\circ} 36^{\prime} 58.6^{\prime \prime}$ & $90^{\circ} 46^{\prime} 37.9^{\prime \prime}$ & Guatemala & Salgado-Maldonado et al. (2014) \\
\hline (47) & Río Cauca (Rcau) & $13^{\circ} 46^{\prime} 42.6^{\prime \prime}$ & $89^{\circ} 51^{\prime} 40.8^{\prime \prime}$ & El Salvador & Salgado-Maldonado et al. (2014) \\
\hline (48) & Río Cauca (Cauc) & $13^{\circ} 46^{\prime} 41.6^{\prime \prime}$ & $89^{\circ} 46^{\prime} 41.67^{\prime \prime}$ & El Salvador & Salgado-Maldonado et al. (2014) \\
\hline (49) & Quebrada Los Tecomates (Qtec) & $14^{\circ} 18^{\prime} 11.3^{\prime \prime}$ & $89^{\circ} 09^{\prime} 40.8^{\prime \prime}$ & El Salvador & Salgado-Maldonado et al. (2014) \\
\hline (50) & Río Nonoalpa (Nono) & $14^{\circ} 17^{\prime} 23.2^{\prime \prime}$ & $89^{\circ} 09^{\prime} 10.7^{\prime \prime}$ & El Salvador & Salgado-Maldonado et al. (2014) \\
\hline (51) & Río Ojojona (Ojoj) & $13^{\circ} 55^{\prime} 43.7^{\prime \prime}$ & $87^{\circ} 17^{\prime} 40^{\prime \prime}$ & Honduras & Salgado-Maldonado et al. (2014) \\
\hline (52) & Lepaterique (Lepa) & $14^{\circ} 03^{\prime} 42.9^{\prime \prime}$ & $87^{\circ} 27^{\prime} 58.6^{\prime \prime}$ & Honduras & Salgado-Maldonado et al. (2014) \\
\hline (53) & Lepateri & $14^{\circ} 04^{\prime} 14.4^{\prime \prime}$ & $87^{\circ} 25^{\prime} 56.9^{\prime \prime}$ & & lgado-Maldonado et al. (2014) \\
\hline (54) & $\begin{array}{l}\text { El Platanar, Putla de Guer } \\
\text { (Plat)* }^{*}\end{array}$ & $16^{\circ} 44^{\prime} 55^{\prime \prime}$ & $32 "$ & México & This study \\
\hline (55) & $\begin{array}{c}\text { Río San José, Santiago Jamiltepec } \\
\text { (Jami)* }\end{array}$ & $16^{\circ} 24^{\prime} 19^{\prime \prime}$ & $97^{\circ} 44^{\prime} 20^{\prime \prime}$ & México & This study \\
\hline (56) & $\begin{array}{c}\text { Río San Juan, Cristobal Obregón } \\
\text { (Obre)* }\end{array}$ & $16^{\circ} 21^{\prime} 00^{\prime \prime}$ & $93^{\circ} 30^{\prime} 54^{\prime \prime}$ & México & This study \\
\hline (57) & $\begin{array}{l}\text { Río Suchiapan, La Esperanza } \\
\text { (Espe)* }\end{array}$ & $16^{\circ} 23^{\prime} 27.60^{\prime \prime}$ & $93^{\circ} 17^{\prime} 24^{\prime \prime}$ & México & This study \\
\hline (58) & Río Pedregal, Tres Picos (Tpic)* & $15^{\circ} 55^{\prime} 1.2^{\prime \prime}$ & $93^{\circ} 32^{\prime} 45.6^{\prime \prime}$ & México & This study \\
\hline (59) & Río Huixtla (Huix)* & $15^{\circ} 10^{\prime} 18^{\prime \prime}$ & $92^{\circ} 25^{\prime} 24^{\prime \prime}$ & México & This study \\
\hline$(60)$ & El Triunfo (Triu)* & $15^{\circ} 20^{\prime} 44^{\prime \prime}$ & $92^{\circ} 32^{\prime} 30^{\prime \prime}$ & México & This study \\
\hline (61) & Río Nueva Francia (Fran)* & $15^{\circ} 22^{\prime} 7.58^{\prime \prime}$ & $92^{\circ} 35^{\prime} 20.2^{\prime \prime}$ & México & $\begin{array}{c}\text { Pérez-Ponce de León et al. (2015) } \\
\text { This study }\end{array}$ \\
\hline (62) & Río Nahualate (Nahu)* & $14^{\circ} 26^{\prime} 44^{\prime \prime}$ & $91^{\circ} 22^{\prime} 56^{\prime \prime}$ & Guatemala & This study \\
\hline (63) & Río Primavera (Prim)* & $14^{\circ} 22^{\prime} 19.20^{\prime \prime}$ & $91^{\circ} 09^{\prime} 60^{\prime \prime}$ & Guatemala & This study \\
\hline (64) & Río Escuintla (Escu)* & $14^{\circ} 19^{\prime} 41.51^{\prime \prime}$ & $91^{\circ} 42^{\prime} 57.35^{\prime \prime}$ & Guatemala & This study \\
\hline (65) & Río Las Cabezas, Saranate (Sara)* & $14^{\circ} 44^{\prime} 23^{\prime \prime}$ & $90^{\circ} 04^{\prime} 52^{\prime \prime}$ & Guatemala & This study \\
\hline (66) & Puente Sansare (Sans)* & $14^{\circ} 44^{\prime} 52^{\prime \prime}$ & $90^{\circ} 06^{\prime} 33^{\prime \prime}$ & Guatemala & This study \\
\hline (67) & Río Hondo (Rhon)* & $15^{\circ} 03^{\prime} 55.50^{\prime \prime}$ & $89^{\circ} 35^{\prime} 48.28^{\prime \prime}$ & Guatemala & This study \\
\hline
\end{tabular}




\begin{tabular}{c|c|c|c|c|c}
\hline \multirow{2}{*}{ CS } & \multirow{2}{*}{ Locality (code) } & \multicolumn{2}{|c|}{ Geographical coordinates } & \multirow{2}{*}{ Country } & \multirow{2}{*}{ References } \\
\cline { 3 - 4 } & & $\mathbf{N}$ & $\mathbf{W}$ & & \multirow{2}{*}{ This study } \\
\hline \multirow{2}{*}{$(68)$} & $\begin{array}{c}\text { Arroyo en Hidroeléctrica } \\
\text { Chamelecón (Cham)* }\end{array}$ & $15^{\circ} 11^{\prime} 51.60^{\prime \prime}$ & $89^{\circ} 36^{\prime} 57.60^{\prime \prime}$ & Honduras & This study \\
\hline$(69)$ & Quebrada El Paraiso (Qpar) & $15^{\circ} 01^{\prime} 26^{\prime \prime}$ & $88^{\circ} 59^{\prime} 32^{\prime \prime}$ & Honduras & This study \\
\hline$(70)$ & Los Potrerillos (Lpot)* & $14^{\circ} 32^{\prime} 31^{\prime \prime}$ & $87^{\circ} 52^{\prime} 55^{\prime \prime}$ & Honduras & This study \\
\hline$(71)$ & Río San Carlos (Rcar) & $16^{\circ} 19^{\prime} 10^{\prime \prime}$ & $91^{\circ} 58^{\prime} 06^{\prime \prime}$ & México & This study \\
\hline$(72)$ & Río La Gloria (Lglo) & $16^{\circ} 30^{\prime} 01^{\prime \prime}$ & $92^{\circ} 26^{\prime} 01^{\prime \prime}$ & México & This study \\
\hline$(73)$ & Arroyo Moxviquil (Moxv)* & $16^{\circ} 54^{\prime} 9.00^{\prime \prime}$ & $92^{\circ} 37^{\prime} 50^{\prime \prime}$ & México & This study \\
\hline$(74)$ & Arroyo Peje de Oro (Poro)* & $16^{\circ} 44^{\prime} 48^{\prime \prime}$ & $92^{\circ} 36^{\prime} 60^{\prime \prime}$ & México & \\
\hline
\end{tabular}

and that the list of helminth parasites of fish of this genus consists of 20 species classified in two taxonomic groups: Platyhelminthes (six adult digeneans, five metacercariae, two monogeneans and one adult cestode) and Nematoda (three adults and three larvae). Interestingly, no acanthocephalans and no larval cestodes are part of the helminth fauna of this fish group across its geographical distribution. Most taxa were identified to species level, except for larval stages which lacked the diagnostic characteristics present only in adult forms which are found in fish-eating birds (or freshwater turtles in the case of Spiroxys sp.). To better visualize the information from the checklist, the results are presented in two tables. Table 2 shows a parasite-host list. Species of parasites are organized by developmental stage, either as adults or larvae, and ordered alphabetically by family name. Species within each family are then listed alphabetically followed by their authority. The host-parasite list (Table 3) is organized alphabetically. Within each fish species, helminth parasites are listed alphabetically by taxonomic group, with their developmental stage indicated in parentheses.

Digeneans exhibit the highest species richness (11 species), followed by nematodes (six species) and monogeneans (two species) (Table 2). Based on the observed hostspecificity, at least six of the 12 adult helminth taxa listed in this work, i.e. Paracreptotrema blancoi sensu Salgado-Maldonado et al. (2011b), P. profundulusi Salgado-Maldonado, Caspeta-Mandujano \& Martínez Ramírez, 2011, Phyllodistomum spinopapillatum Pérez-Ponce de León, Pinacho-Pinacho, Mendoza-Garfias \& García-Varela, 2015, Spinitectus humbertoi Mandujano-Caspeta \& Moravec, 2000, S. mariaisabelae Caspeta-Mandujano Cabañas-Carranza \& Salgado-Maldonado, 2007 and Rhabdochona salgadoi Mandujano-Caspeta \& Moravec, 2000, have only been recorded as parasites of profundulids and can be considered as members of the 'core' helminth fauna (in an historical biogeographical sense, not to be confused with the ecological "coresatellite" species concept, see Pérez-Ponce de León and Choudhury 2002). The cestode Bothriocephalus acheilognathi Yamaguti, 1934 has successfully infected some species of Profundulus; this is an introduced species that is commonly found in several freshwater fishes in North and Central America as a result of the introduction of cyprinids (carps) for aquaculture (see Choudhury et al. 2013). The digenean Centrocestus formosanus Nishigori, 1924 also represents a species that was introduced in North America, and perhaps Middle-America, through the introduction of its snail host, Melanoides tuber- 


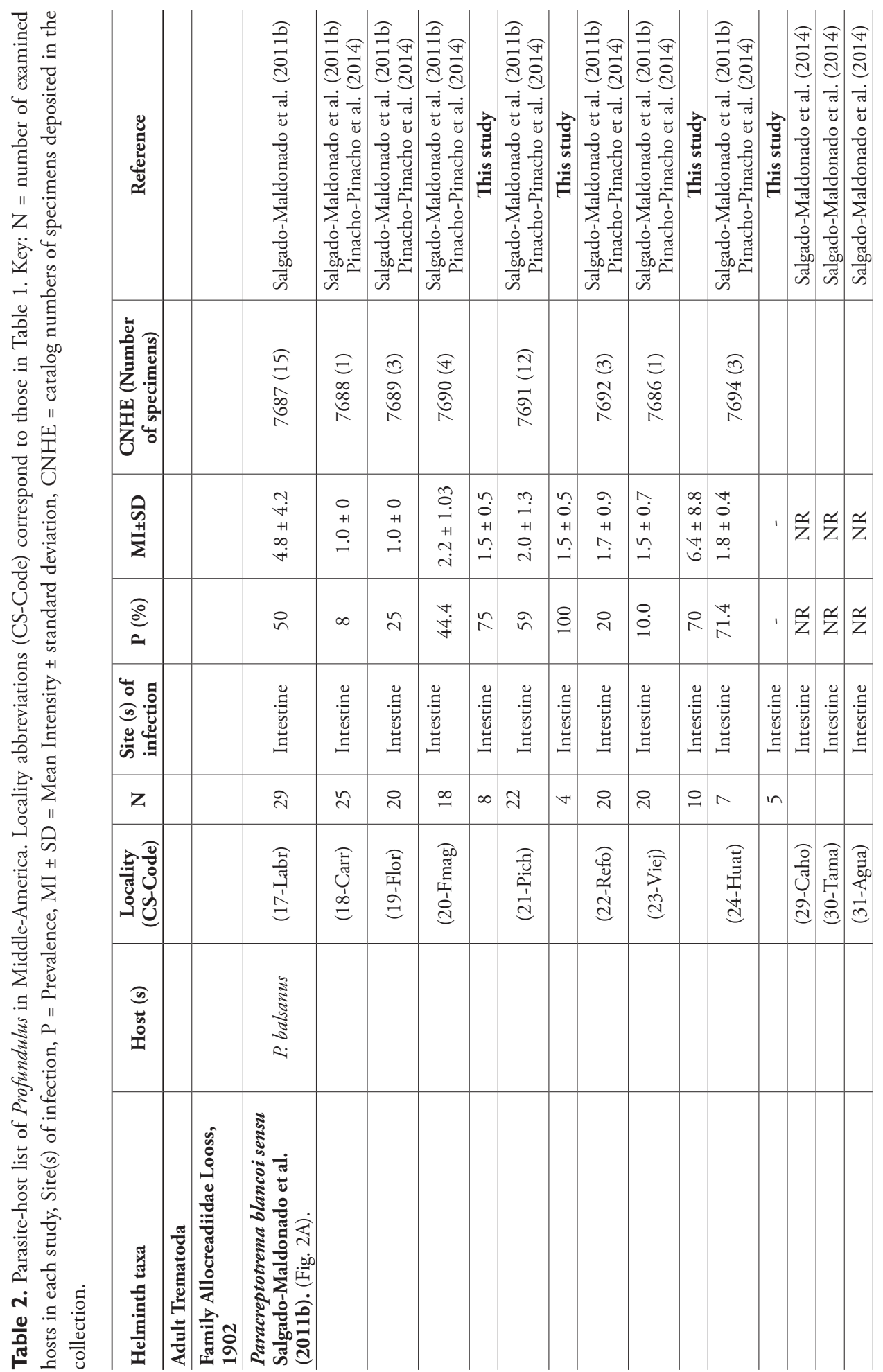




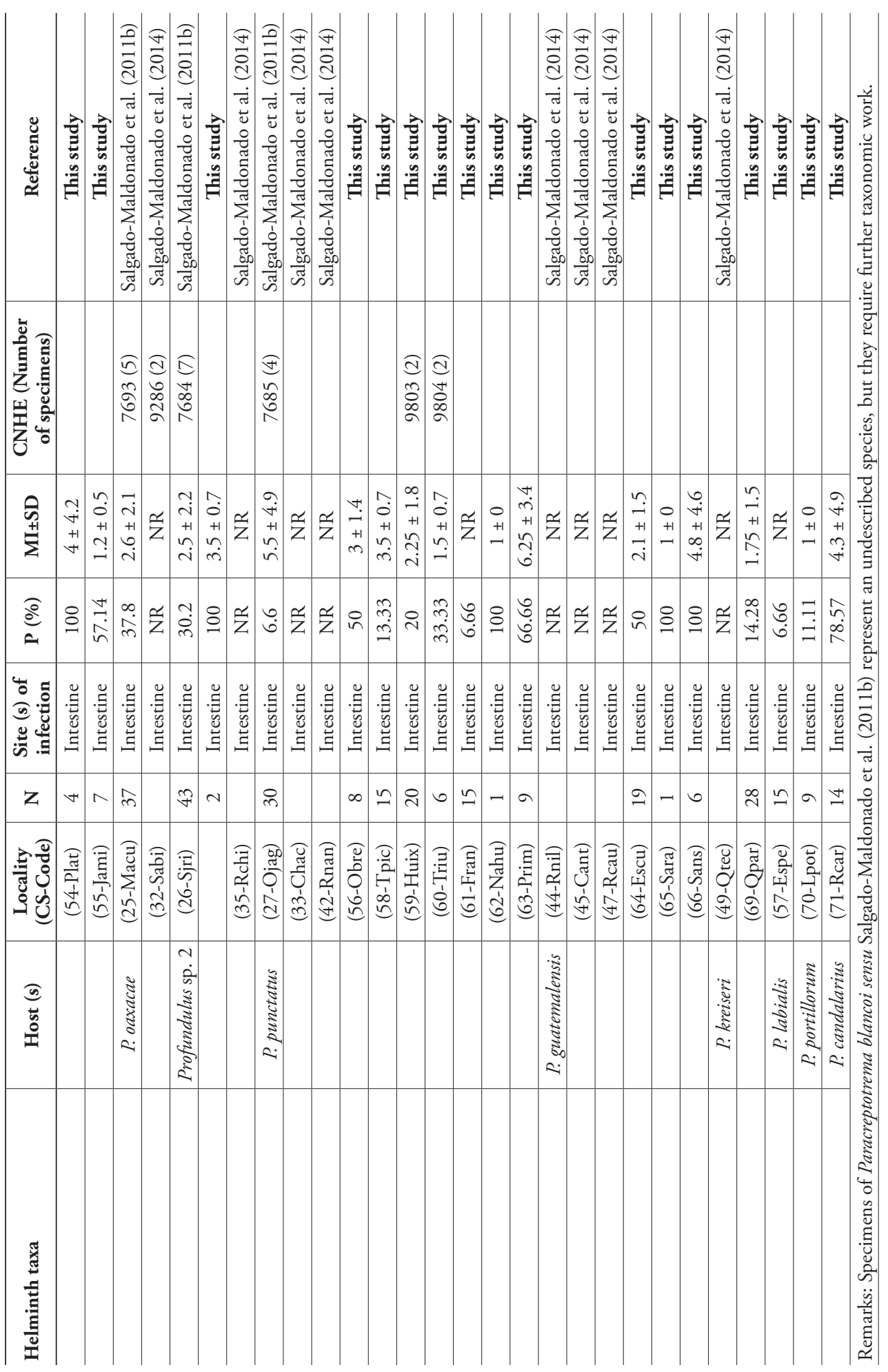




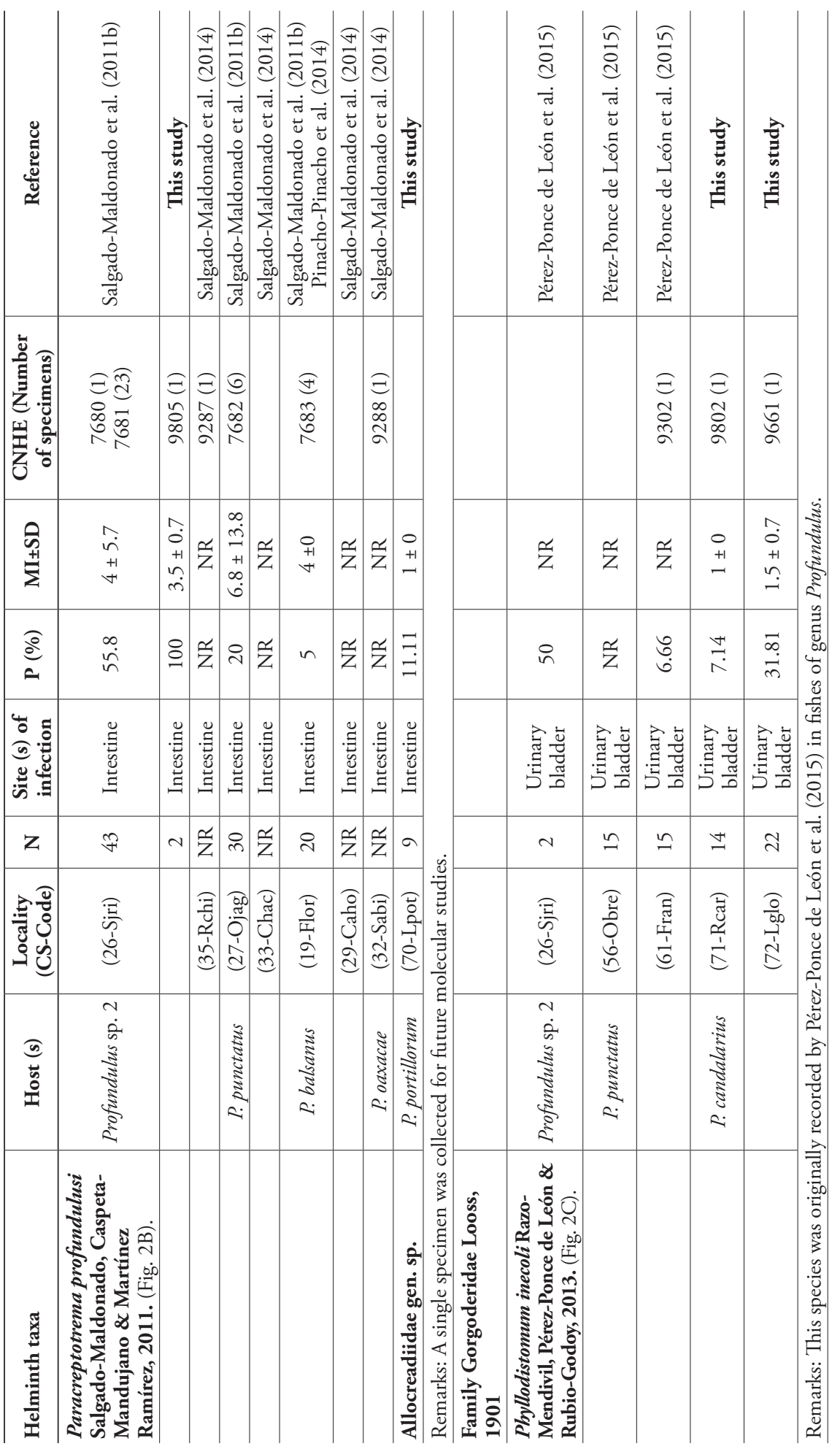




\begin{tabular}{|c|c|c|c|c|c|c|c|c|c|c|c|c|c|c|}
\hline 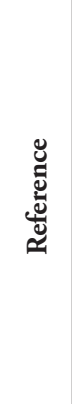 & 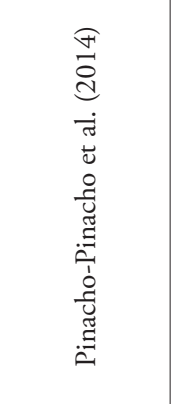 & 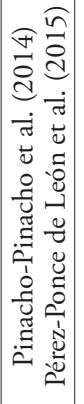 & $\begin{array}{l}\overrightarrow{\vec{z}} \\
\vec{E} \\
\stackrel{0}{E} \\
\vec{F}\end{array}$ & 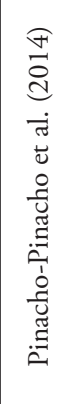 & 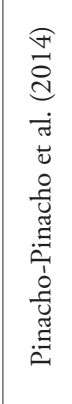 & 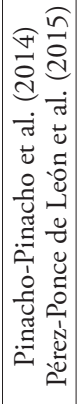 & 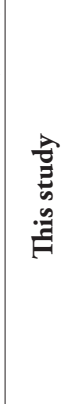 & 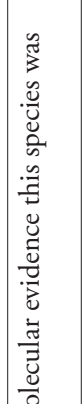 & & 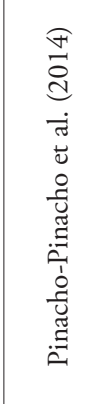 & 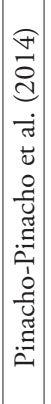 & 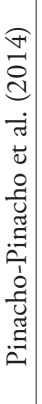 & 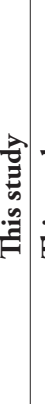 & 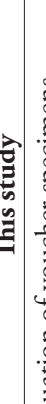 \\
\hline 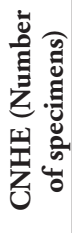 & 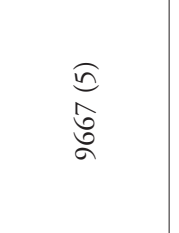 & 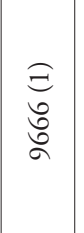 & $\begin{array}{l}\Xi \\
\overrightarrow{\widehat{\sigma}} \\
\curvearrowright\end{array}$ & $\begin{array}{l}\Xi \\
\stackrel{0}{0} \\
\stackrel{0}{0}\end{array}$ & & 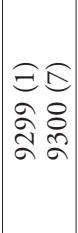 & & 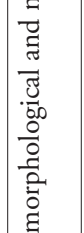 & & 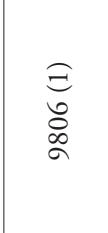 & & & & \\
\hline $\begin{array}{l}\text { 会 } \\
\text { +1 } \\
\sum\end{array}$ & $\stackrel{+}{+1}$ & $\begin{array}{l}0 \\
+1 \\
-1\end{array}$ & $\begin{array}{l}0 \\
+1 \\
-\end{array}$ & $\begin{array}{l}\text { + } \\
+1 \\
-\end{array}$ & 운 & 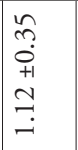 & $\begin{array}{l}n \\
\dot{0} \\
+1 \\
\infty \\
-\end{array}$ & 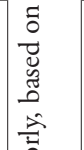 & & $\begin{array}{l}\stackrel{n}{a} \\
\stackrel{+}{+1} \\
\stackrel{n}{n} \\
\dot{n}\end{array}$ & \begin{tabular}{c|}
$\infty$ \\
$\infty$ \\
0 \\
+1 \\
\\
\\
\end{tabular} & $\begin{array}{l}\text { O } \\
+1 \\
-\end{array}$ & $\begin{array}{l}0 \\
+1 \\
+1\end{array}$ & 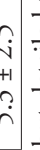 \\
\hline 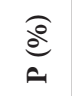 & ㄱ & $\left\{\begin{array}{l}\hat{n} \\
\hat{n}\end{array}\right.$ & $\approx$ & $\stackrel{\forall}{\stackrel{*}{*}}$ & $\stackrel{0}{ }$ & 우 & $R$ & 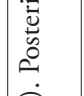 & & 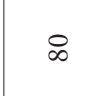 & in & $\begin{array}{l}\Xi \\
\Xi \\
\Xi\end{array}$ & $\stackrel{n}{i}$ & $\begin{array}{l}6 \\
6 \\
\dot{y} \\
\end{array}$ \\
\hline 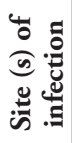 & 氧泀 & 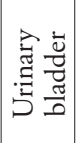 & 醇 & 急泀 & 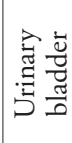 & 豙节 & 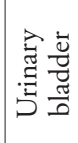 & 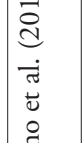 & & 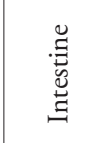 & 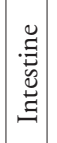 & 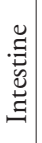 & 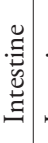 & : \\
\hline z & $\approx$ & $\stackrel{\infty}{\sim}$ & $\infty$ & $\tilde{N}$ & $\stackrel{\lambda}{ }$ & $\stackrel{\text { i }}{ }$ & $\therefore$ & $\stackrel{\Xi}{\Xi}$ & & $\approx$ & i & $\stackrel{\infty}{\sim}$ & $\infty$ & C \\
\hline 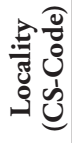 & $\begin{array}{l}\text { : } \\
\bigcup_{1} \\
\stackrel{0}{=}\end{array}$ & 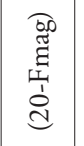 & & 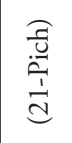 & 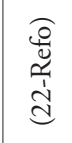 & 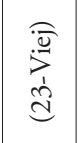 & & 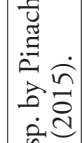 & & 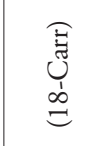 & $\begin{array}{l}\overparen{\overrightarrow{0}} \\
\frac{\vec{D}}{I I} \\
\stackrel{\Xi}{\Xi}\end{array}$ & 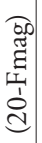 & & $\begin{array}{l}\widehat{a} \\
\frac{\pi}{1} \\
\hat{n}\end{array}$ \\
\hline 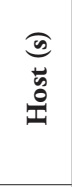 & $\begin{array}{l}\frac{3}{3} \\
\frac{3}{3} \\
\frac{3}{5} \\
\text { ci }\end{array}$ & & & & & & & 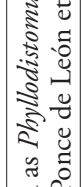 & & $\begin{array}{l}3 \\
\frac{3}{3} \\
\frac{3}{3} \\
\text { a }\end{array}$ & & & & \\
\hline 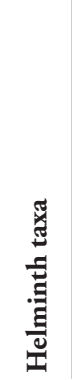 & 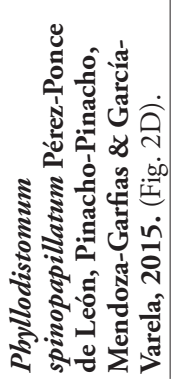 & & & & & & & 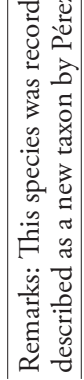 & 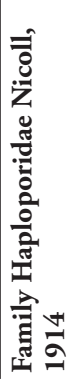 & 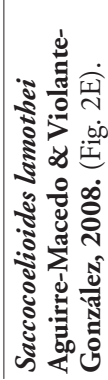 & & & & \\
\hline
\end{tabular}




\begin{tabular}{|c|c|c|c|c|c|c|c|c|c|c|c|c|c|c|}
\hline 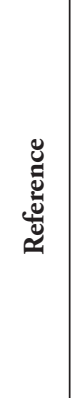 & & & 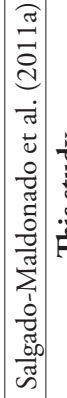 & 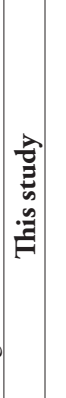 & 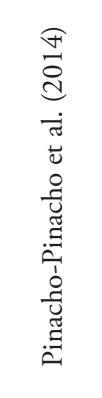 & 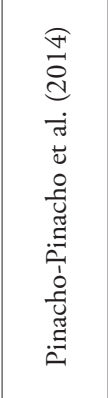 & 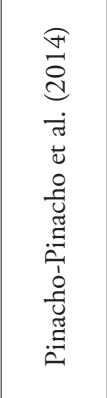 & 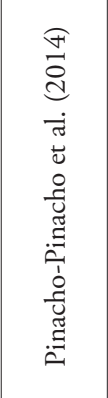 & 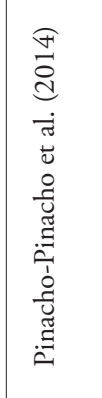 & 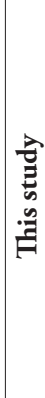 & 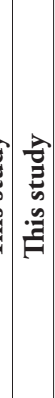 & 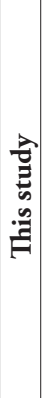 & 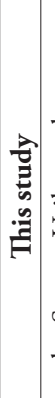 & 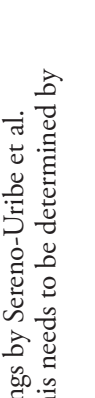 \\
\hline 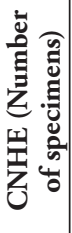 & & & 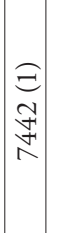 & & 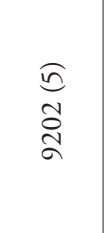 & & & & & & & $\begin{array}{l}\Xi \\
0 \\
0 \\
0 \\
2\end{array}$ & & 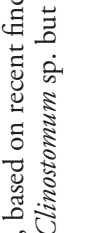 \\
\hline $\begin{array}{l}2 \\
\infty \\
+1 \\
\sum\end{array}$ & & & $\begin{array}{c}0 \\
\dot{0} \\
\pm 1 \\
-1\end{array}$ & 孚 & $\begin{array}{l}\stackrel{+}{\sigma} \\
\stackrel{+}{+1} \\
\stackrel{i}{i}\end{array}$ & $\begin{array}{l}\text { O+ } \\
+\end{array}$ & $\begin{array}{l}0 \\
+1 \\
-\end{array}$ & $\begin{array}{l}0 \\
0 \\
+1 \\
n \\
\end{array}$ & $\begin{array}{l}0 \\
+1 \\
-1\end{array}$ & 号 & $\mid \begin{array}{l}0 \\
+1 \\
n\end{array}$ & $\begin{array}{c}0 \\
+1 \\
-1\end{array}$ & $\begin{array}{c}0 \\
+1 \\
\sim\end{array}$ & 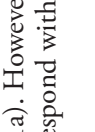 \\
\hline $\begin{array}{c}\grave{e} \\
a\end{array}$ & & & $\begin{array}{cc}n \\
\infty\end{array}$ & 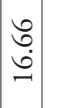 & 아 & $\begin{array}{l}n \\
\tilde{n} \\
n\end{array}$ & 으 & 으 & $\begin{array}{l}\approx \\
\infty \\
\stackrel{f}{\forall}\end{array}$ & 党 & $\approx$ & 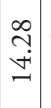 & 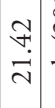 & 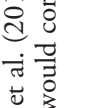 \\
\hline 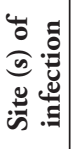 & & & 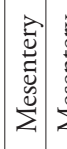 & 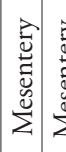 & 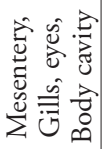 & 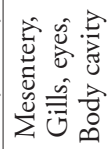 & 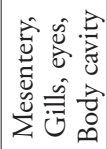 & 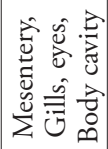 & 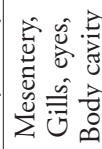 & 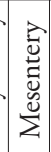 & 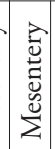 & 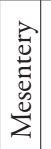 & : & 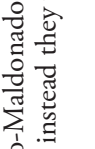 \\
\hline z & & & $\approx v$ & ○ & $\approx$ & $\stackrel{\infty}{-}$ & 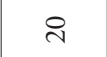 & ㄱ. & $\wedge$ & $n$ & $\psi$ & $\curvearrowright$ & $\Xi$ & $\pi 0$ \\
\hline 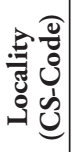 & & & 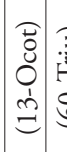 & 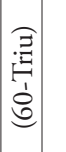 & $\begin{array}{l}\text { E्ञ } \\
\bigcup_{1} \\
\stackrel{0}{=}\end{array}$ & 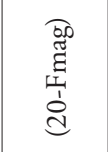 & $\begin{array}{l}\text { ô } \\
\stackrel{\Delta}{1} \\
\stackrel{d}{d} \\
\text { d }\end{array}$ & $\begin{array}{l}\stackrel{\widehat{\vartheta}}{9} \\
\stackrel{1}{d} \\
\stackrel{d}{d}\end{array}$ & 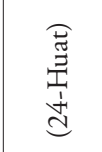 & & 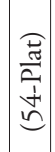 & 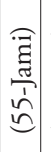 & 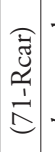 & 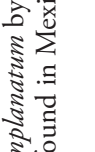 \\
\hline $\begin{array}{l}\widehat{B} \\
\stackrel{0}{0} \\
\stackrel{0}{1}\end{array}$ & & & 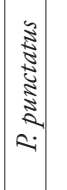 & & $\begin{array}{l}3 \\
\text { s. } \\
\frac{3}{3} \\
\frac{1}{2} \\
\text { ai }\end{array}$ & & & & & & & & 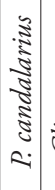 & 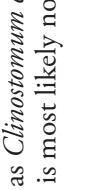 \\
\hline 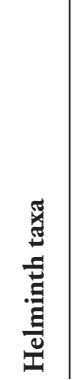 & 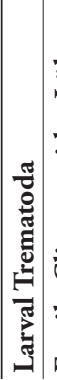 & 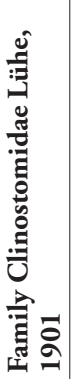 & 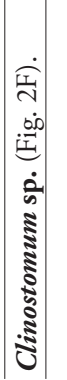 & & & & & & & & & & & 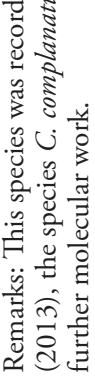 \\
\hline
\end{tabular}




\begin{tabular}{|c|c|c|c|c|c|c|c|c|c|c|c|c|c|c|c|}
\hline 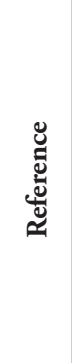 & & 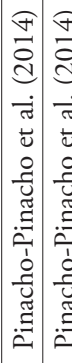 & 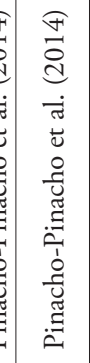 & 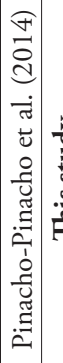 & 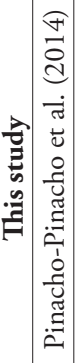 & 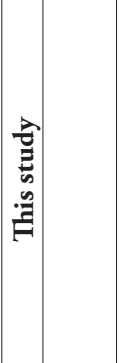 & 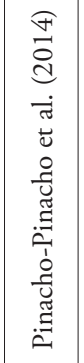 & 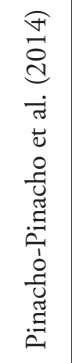 & 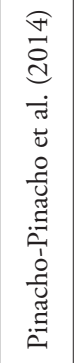 & 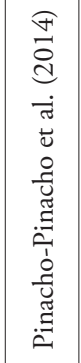 & 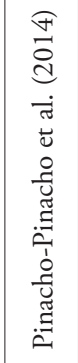 & 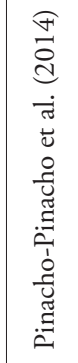 & 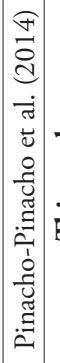 & 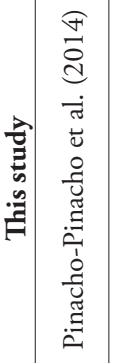 & 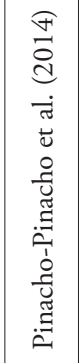 \\
\hline 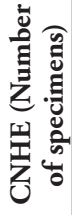 & & & & & 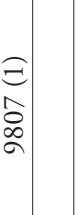 & & $\begin{array}{l}\stackrel{\sigma}{\sigma} \\
\stackrel{\sigma}{\sigma}\end{array}$ & & & $\begin{array}{l}\text { () } \\
\text { \& } \\
\text { a }\end{array}$ & & & & & $\begin{array}{l}\widehat{\Omega} \\
\text { స్ } \\
\text { a }\end{array}$ \\
\hline $\begin{array}{l}\text { 忩 } \\
+1 \\
\sum_{2}^{+1}\end{array}$ & & 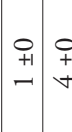 & 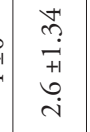 & \begin{tabular}{l|l}
0 & 0 \\
+1 & 0 \\
- & 0
\end{tabular} & \begin{tabular}{l|l|}
0 & 0 \\
+1 & ++1 \\
$N$ & $\forall$
\end{tabular} & 孚 & 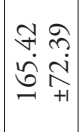 & 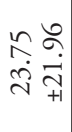 & $\begin{array}{cc}n & 0 \\
\hat{\sigma} & 0 \\
- & \stackrel{n}{+1}\end{array}$ & 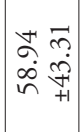 & 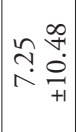 & 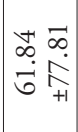 & $\begin{array}{ll}0 & 1 \\
+1 & 5\end{array}$ & 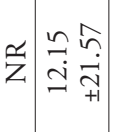 & 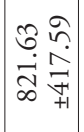 \\
\hline$e^{a}$ & & $\forall v$ & $\approx$ & $\operatorname{in}$ & 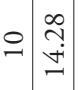 & 号 & $\stackrel{\infty}{\sim}$ & 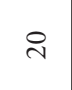 & $\begin{array}{l}n \\
\infty \\
\infty\end{array}$ & 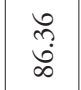 & 8 & $\curvearrowleft$ & 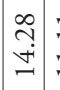 & $\begin{array}{l}\exists \\
\Xi \\
\Xi \\
\end{array}$ & 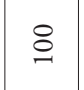 \\
\hline 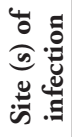 & & 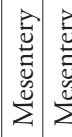 & 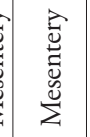 & 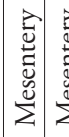 & 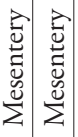 & 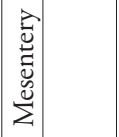 & 节 & 节 & 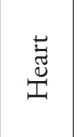 & 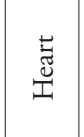 & 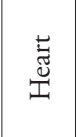 & 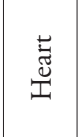 & : & 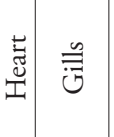 & 氙 \\
\hline z & & $\approx$ & iิ & 교 & $\therefore \wedge$ & $n$ & 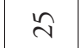 & ㄱ. & $\stackrel{\infty}{-}$ & ปี & i & i & $\wedge$ & $\stackrel{\infty}{\sim}$ & ปี \\
\hline 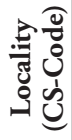 & & 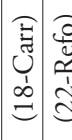 & 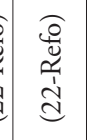 & 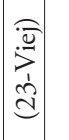 & 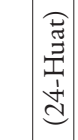 & & $\begin{array}{l}\overparen{E} \\
\stackrel{0}{0} \\
\infty \\
=\end{array}$ & $\begin{array}{l}\overparen{\overrightarrow{0}} \\
\text { II } \\
\stackrel{1}{\Xi}\end{array}$ & 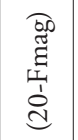 & 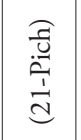 & $\begin{array}{l}\text { Q } \\
\stackrel{2}{1} \\
\text { d }\end{array}$ & 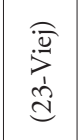 & 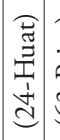 & 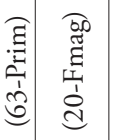 & 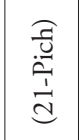 \\
\hline 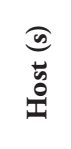 & & 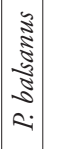 & $\begin{array}{l}3 \\
3 \\
\frac{3}{3} \\
\frac{3}{8} \\
\text { a }\end{array}$ & & & & 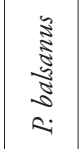 & & & & & & & 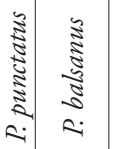 & \\
\hline 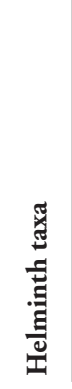 & 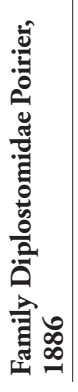 & 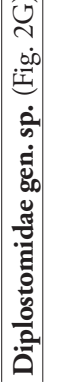 & 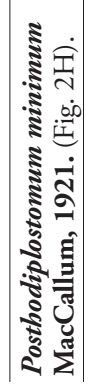 & & & 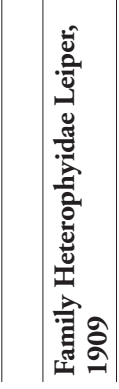 & 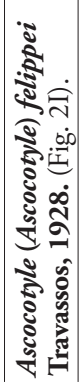 & & & & & & & 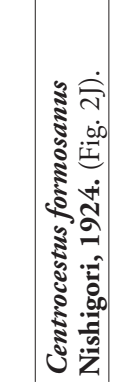 & \\
\hline
\end{tabular}




\begin{tabular}{|c|c|c|c|c|c|c|c|c|c|c|c|c|c|c|c|c|c|}
\hline 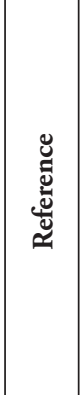 & 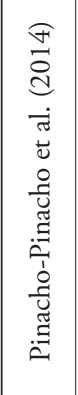 & 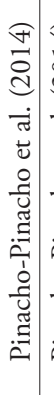 & 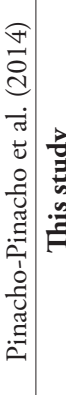 & 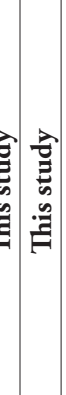 & & 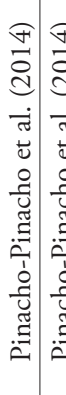 & 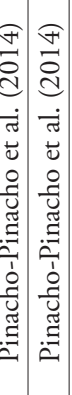 & 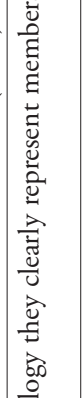 & & 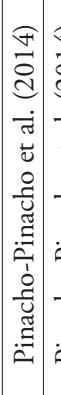 & 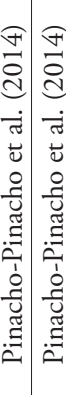 & 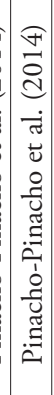 & 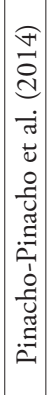 & 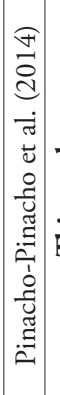 & 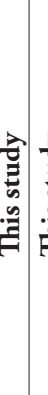 & 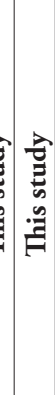 & 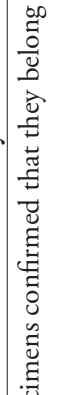 \\
\hline 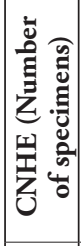 & & & & & & & & 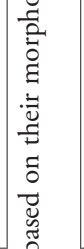 & & & & & & & & & 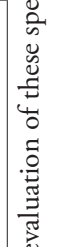 \\
\hline $\begin{array}{l}\text { O } \\
\text { 立 } \\
\sum_{\Sigma}\end{array}$ & 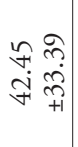 & $\begin{array}{l}0 \\
+1 \\
\bar{m}\end{array}$ & 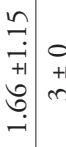 & $\begin{array}{l}D_{+1}^{+} \\
\end{array}$ & & \begin{tabular}{c|c} 
& 0 \\
+1 & + \\
- & 9
\end{tabular} & \begin{tabular}{l|l}
0 & 0 \\
+1 & +1 \\
$\sim$ & -
\end{tabular} & 氞 & & O & 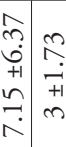 & $\begin{array}{l}n \\
f \\
\sim \\
\sim \\
\sim \\
\sim\end{array}$ & $\begin{array}{c}\hat{y} \\
i \\
+1 \\
i \\
0 \\
0 \\
i n\end{array} \mid$ & 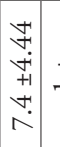 & +1 & +1 & $\begin{array}{l}\overrightarrow{\widetilde{J}} \\
\frac{0}{00} \\
\frac{0}{0} \\
\frac{1}{2} \\
\frac{\pi}{2}\end{array}$ \\
\hline$\frac{\mathfrak{o}}{a}$ & 8 & $\begin{array}{l}\infty \\
\infty \\
\infty \\
\end{array} \mid$ & ๑ & $\vec{Z}$ & & $\operatorname{in} n$ & $n=0$ & 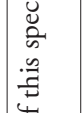 & & $n$ & 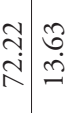 & 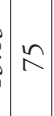 & $\left|\begin{array}{c}n \\
\tilde{j} \\
\infty\end{array}\right|$ & $\begin{array}{c}\infty \\
\tilde{\infty} \\
\infty\end{array}$ & 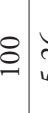 & 8 & 苛 \\
\hline 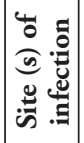 & $\stackrel{\overrightarrow{0}}{\overline{0}}$ & 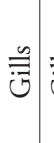 & 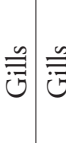 & $\frac{0}{\overline{0}}$ & & 象起 & 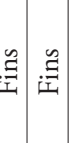 & 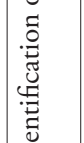 & & 氞 & 㟢击 & 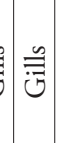 & 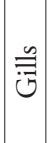 & $\overline{\overrightarrow{0}}$ & $\stackrel{0}{\overline{\vec{j}}} \overline{\bar{j}}$ & $\frac{n}{\overrightarrow{0}}$ & 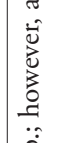 \\
\hline Z & 로 & ¿ & $\wedge-$ & $a$ & & ¿ & 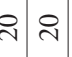 & ب̆ & & ㄱ. & $\stackrel{\infty}{\sim}$ & 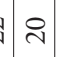 & ิㅏ & $\wedge$ & -9 & - & ב \\
\hline 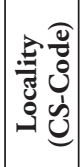 & 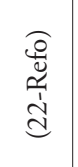 & 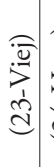 & 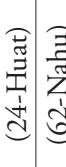 & 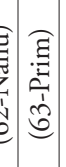 & & 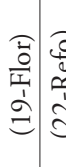 & 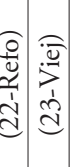 & 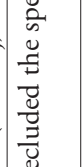 & & 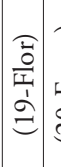 & 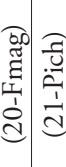 & 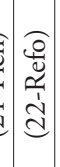 & 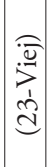 & 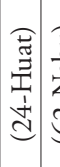 & 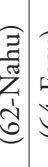 & 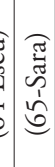 & 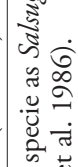 \\
\hline 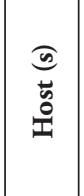 & & & $\begin{array}{c}3 \\
0 \\
0 \\
0 \\
3 \\
0 \\
0 \\
0\end{array}$ & & & 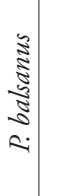 & . & 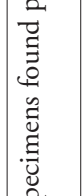 & & $\mid \begin{array}{l}3 \\
z \\
z \\
\tilde{z} \\
z \\
z \\
i \\
i\end{array}$ & & & & & 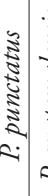 & & 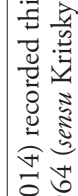 \\
\hline 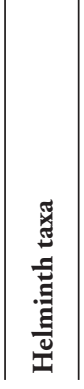 & & & & & 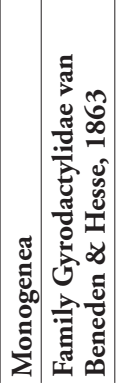 & 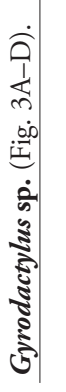 & & 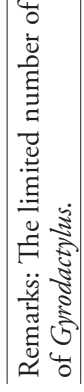 & 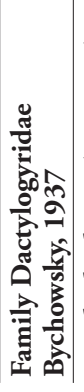 & 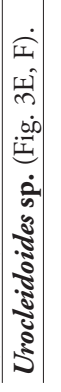 & & & & & & & 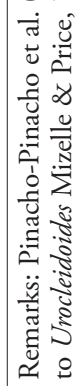 \\
\hline
\end{tabular}




\begin{tabular}{|c|c|c|c|c|c|c|c|c|c|c|c|c|c|c|c|c|c|c|c|c|c|}
\hline 芯 & & & 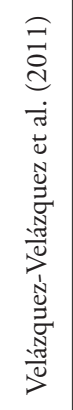 & 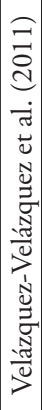 & 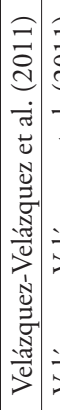 & 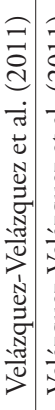 & 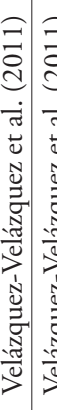 & 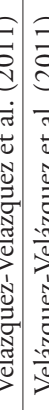 & 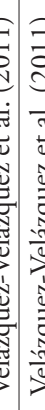 & 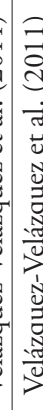 & 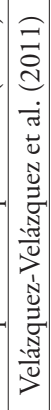 & 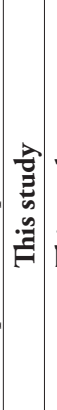 & 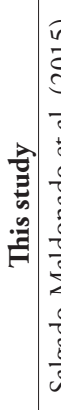 & 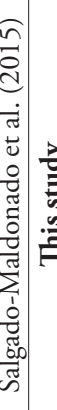 & 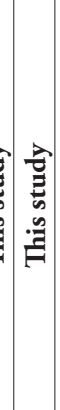 & 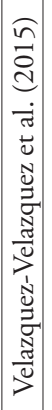 & 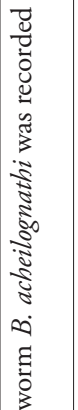 & & & 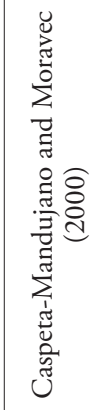 & 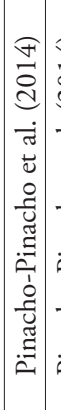 \\
\hline 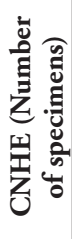 & & & 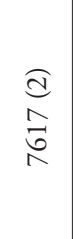 & & & & & & & & & & & 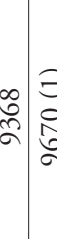 & 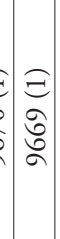 & & 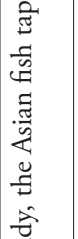 & & & 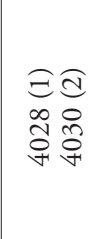 & \\
\hline $\begin{array}{l}\text { Q } \\
\text { +1 } \\
\sum^{+1}\end{array}$ & & & 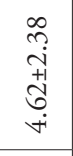 & $\mid$\begin{tabular}{c|}
$\hat{n}$ \\
$\infty$ \\
0 \\
0 \\
0 \\
$-\infty$ \\
\\
\end{tabular} & \begin{tabular}{|c|c}
$\vec{n}$ \\
$\tilde{i}$ \\
+1 \\
$\tilde{n}$ \\
$\tilde{n}$ \\
$\dot{v}$ \\
\end{tabular} & 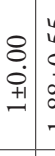 & 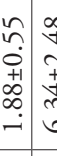 & 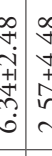 & 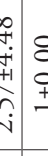 & 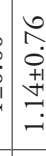 & 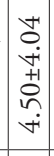 & $\mid \begin{array}{c}a \\
a \\
+1 \\
=\end{array}$ & 孚妾 & $\begin{array}{l}\mathbf{z} \\
\mathbf{z} \\
\forall\end{array}$ & 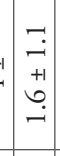 & 吕 & 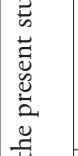 & & & 吕 & $\mid \begin{array}{c}n \\
n \\
\tilde{n} \\
+1 \\
\tilde{n} \\
\tilde{0}\end{array}$ \\
\hline $\begin{array}{l}\stackrel{a}{e} \\
a\end{array}$ & & & $\stackrel{i n}{n}$ & 5 & $\tilde{n}$ & $N=$ & $\vec{F}$ & $R=$ & \pm 0 & on & 更 & ㄱ. & $\begin{array}{l}\sim \\
\infty \\
i \\
f\end{array}$ & $\stackrel{n}{z}$ & $\begin{array}{c}\stackrel{N}{N} \\
\widetilde{N}\end{array}$ & 孚 & 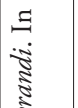 & & & 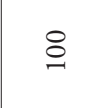 & 으 \\
\hline 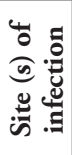 & & & 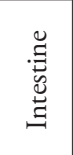 & 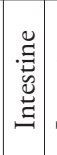 & 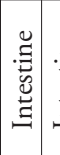 & 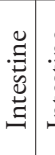 & 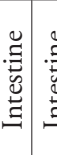 & 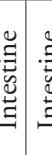 & 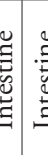 & 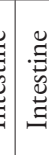 & 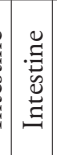 & 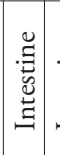 & 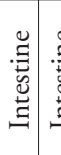 & 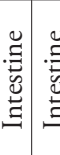 & 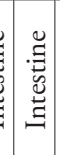 & 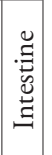 & $\begin{array}{l}\overrightarrow{3} \\
\bar{E} \\
. \Xi \\
\Xi\end{array}$ & & & 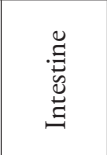 & 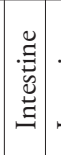 \\
\hline $\mathrm{Z}$ & & & $\stackrel{\sim}{\sim}$ & $\begin{array}{l}\infty \\
0 \\
-\end{array}$ & $\stackrel{m}{=}$ & $\infty$ & 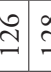 & 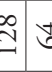 & t) & 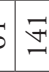 & 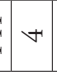 & ¿ & $\wedge$ & ¿ & ปี & 孚 & 离 & & & - & ¿ \\
\hline 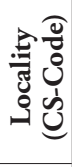 & & & 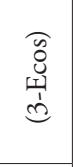 & 焉 & 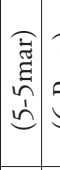 & 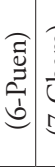 & 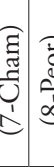 & 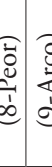 & 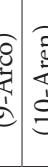 & 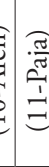 & 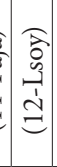 & 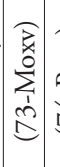 & 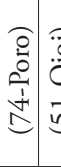 & 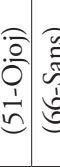 & 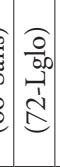 & 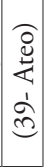 & 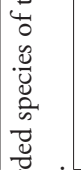 & & & $\begin{array}{l}\text { Oñ } \\
\stackrel{\Xi}{\Xi} \\
\Xi\end{array}$ & 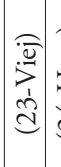 \\
\hline 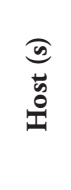 & & & 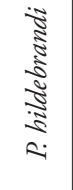 & & & & & & & & & & & 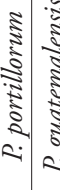 & 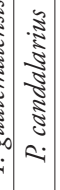 & & 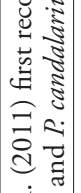 & & & 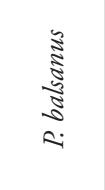 & \\
\hline 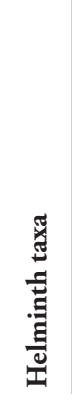 & 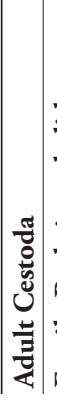 & 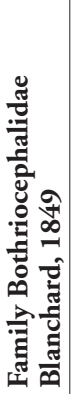 & 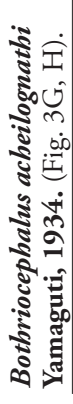 & & & & & & & & & & & & & & 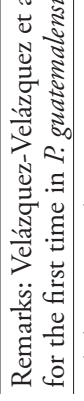 & 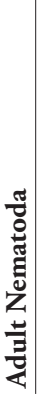 & 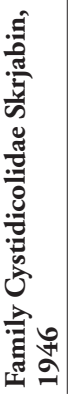 & 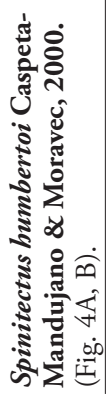 & \\
\hline
\end{tabular}




\begin{tabular}{|c|c|c|c|c|c|c|c|c|c|c|c|c|c|c|c|c|c|c|c|c|}
\hline 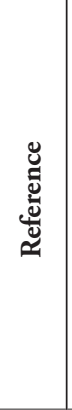 & 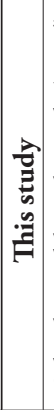 & 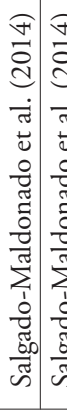 & 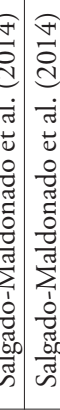 & 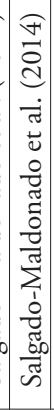 & 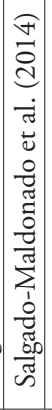 & 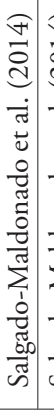 & 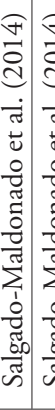 & 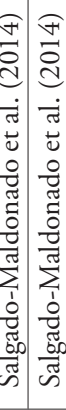 & 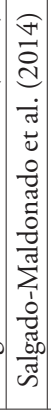 & 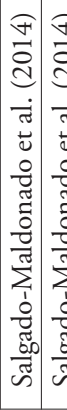 & 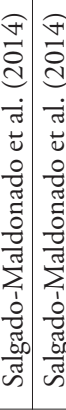 & 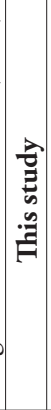 & 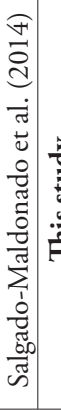 & 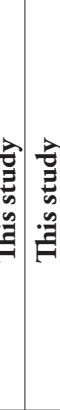 & 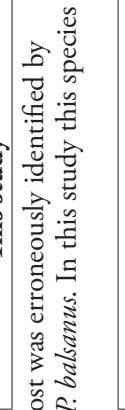 & 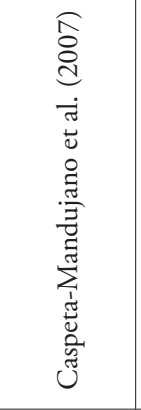 & 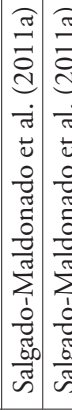 & 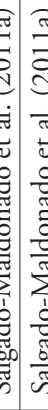 & 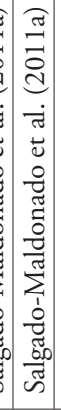 & \\
\hline 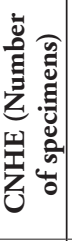 & 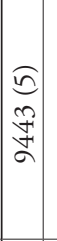 & & & & & & & & & & & $\begin{array}{l}\sqrt{n} \\
\text { ते } \\
\text { }\end{array}$ & 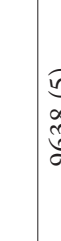 & & 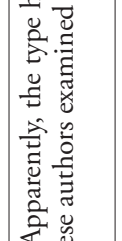 & 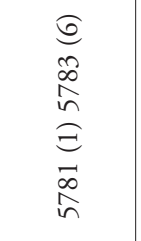 & & & & \\
\hline $\begin{array}{l}2 \\
\text { 市 } \\
\sum^{+1}\end{array}$ & 孚 & 孚孚 & $\frac{r}{z} \mid \frac{r}{z}$ & 孚 & 孚 & 妾 & 孚主 & $\begin{array}{c}\mathbf{z} \\
\mathbf{z}\end{array}$ & 号 & 孚孚 & 孚 & $\left|\begin{array}{c}n \\
+1 \\
m \\
m \\
m\end{array}\right|$ & 点至 & $\begin{array}{l}n \\
\sim \\
i \\
+1 \\
b \\
i\end{array}$ & 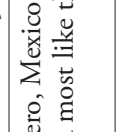 & 点 & 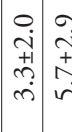 & & 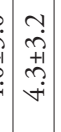 & \\
\hline$\frac{a}{a}$ & 点 & 孚告 & z & 孚 & 孚 & 点 & 孚 & 点 & $\stackrel{\widetilde{z}}{\mathrm{z}}$ & 孚告 & 点 $\mid \stackrel{a}{z}$ & in & 孚 & 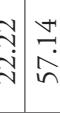 & 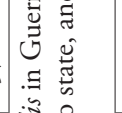 & 㟔 & ¿ & $\stackrel{8}{b}$ & $\frac{1}{2}$ & \\
\hline 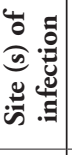 & 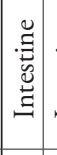 & 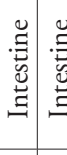 & 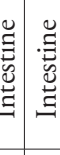 & 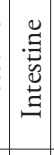 & 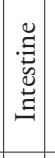 & 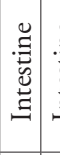 & 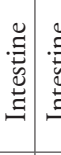 & 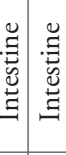 & 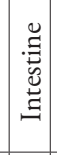 & 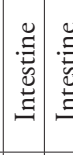 & 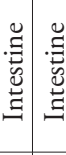 & 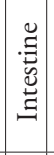 & 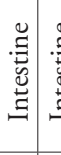 & 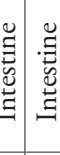 & 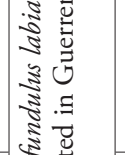 & 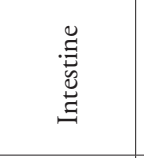 & 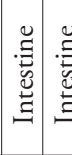 & & 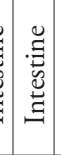 & \\
\hline $\mathrm{z}$ & in & 觉咅 & 光 & 号 & 孚 & 孚 & 岂孚 & 艺 & 吕 & 妾告 & 孚觉 & $\bullet$ & 孚。 & $\curvearrowright \pm$ & & 吕 & $m=$ & $\begin{array}{c}v \\
y\end{array}$ & $\stackrel{\sim}{\sim}$ & $n$ \\
\hline 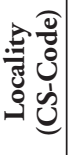 & & 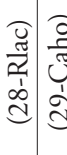 & 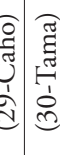 & 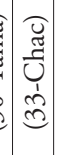 & 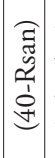 & 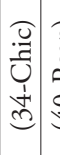 & 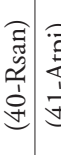 & 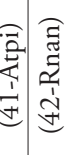 & 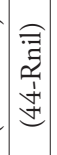 & 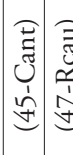 & 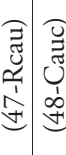 & 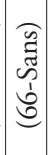 & \begin{tabular}{l|l} 
& \\
0 & \\
0 & \\
$z$ & 1 \\
0 & 1 \\
0 & 1
\end{tabular} & 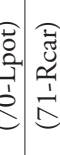 & 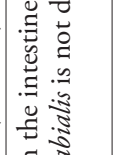 & 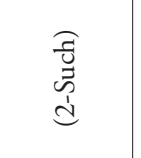 & 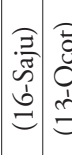 & & 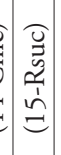 & \\
\hline 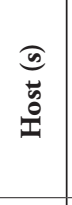 & & & & 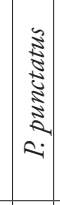 & & 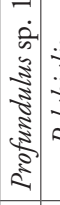 & $\begin{array}{l}\text { a } \\
\text { है } \\
\text { है } \\
\end{array}$ & & 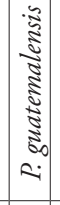 & & & & 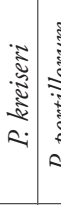 & 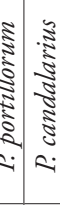 & 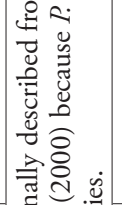 & 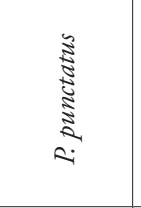 & & है & & \\
\hline 迹 & & & & & & & & & & & & & & & 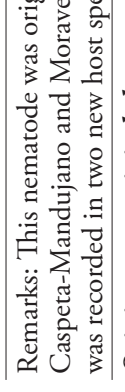 & 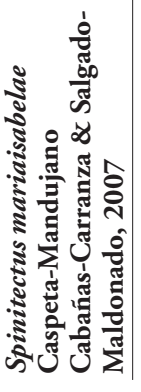 & & & & \\
\hline
\end{tabular}




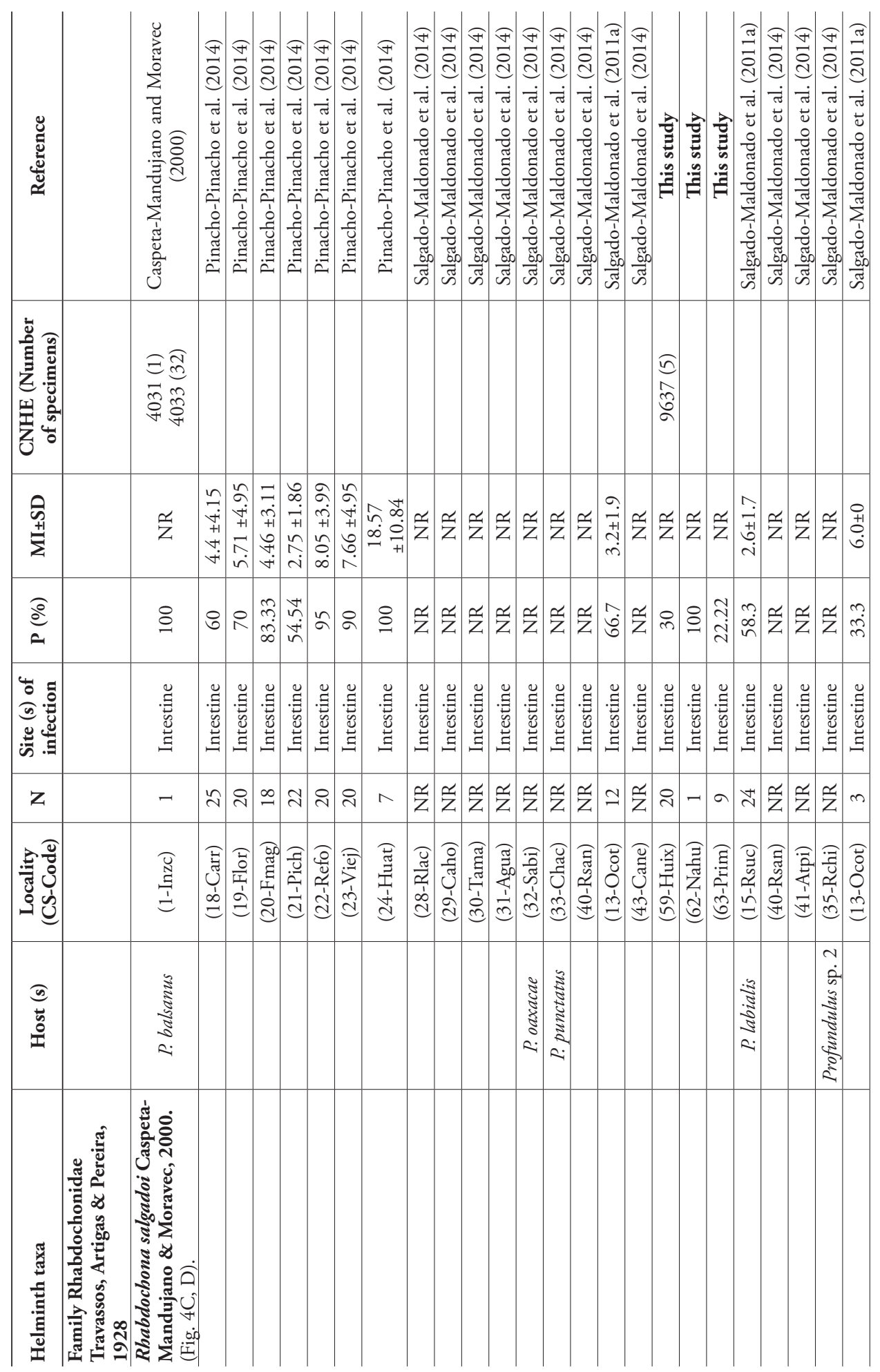




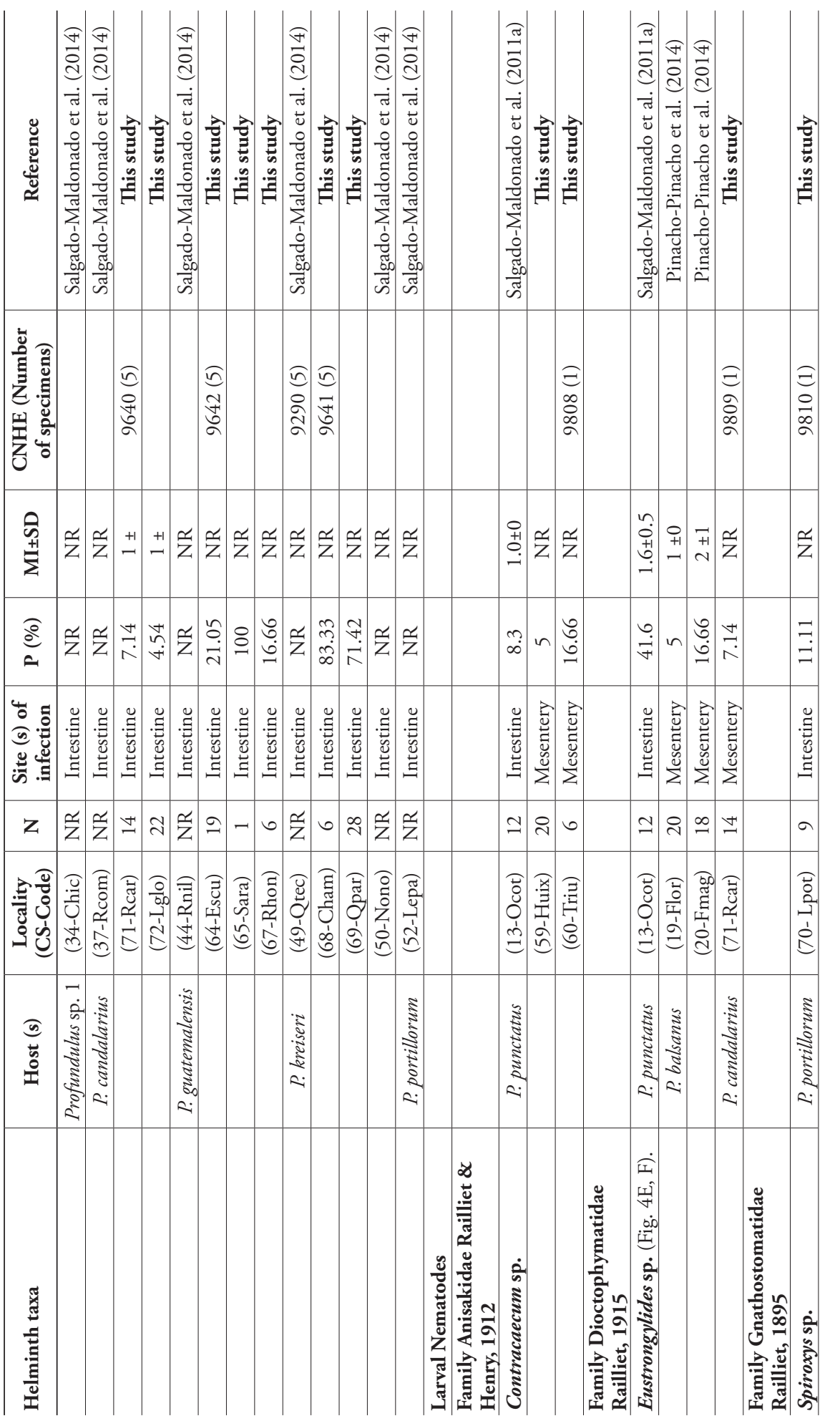




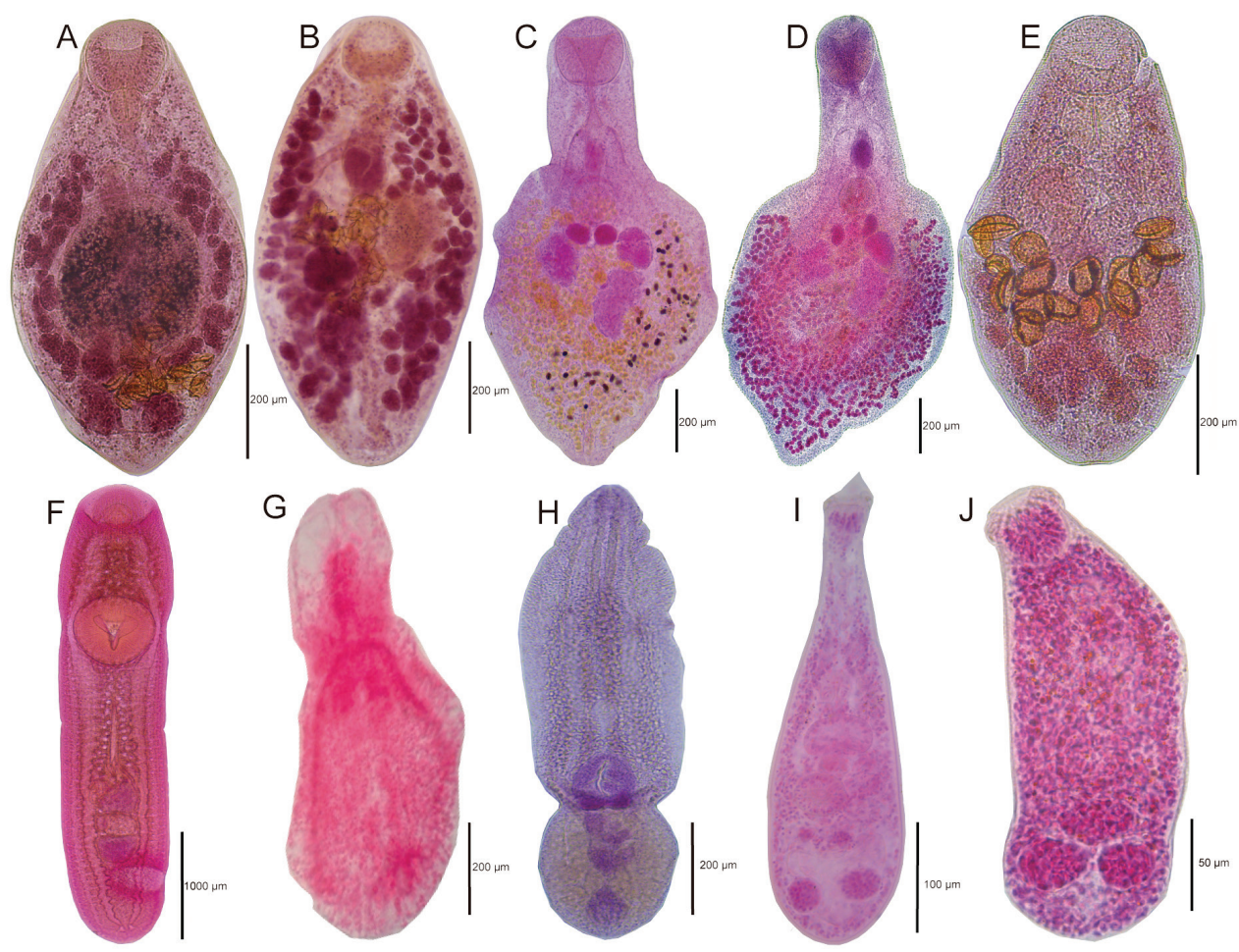

Figure 2. Species of trematodes found in Profundulus spp. A Paracreptotrema blancoi sensu SalgadoMaldonado et al. (2011b) B Paracreptotrema profundulusi C Phyllodistomum inecoli D Phyllodistomum spinopapillatum E Saccocoelioides lamothei F Clinostomum sp. G Diplostomidae gen. sp. H Posthodiplostomum minimum I Ascocotyle (Ascocotyle) felippei $\mathbf{J}$ Centrocestus formosanus.

culata (Müller, 1774), from Asia (Scholz and Salgado-Maldonado 2000). Of the 20 taxa found, eight (40\%) were larval forms of generalist species that use freshwater fish as intermediate or paratenic hosts. In seven of the eight species, fish-eating birds are the definitive hosts, and only one, Spiroxys sp., requires a different vertebrate to complete its life cycle. Adult nematodes of the genus Spiroxys Schneider, 1866 commonly occur in the digestive tract of freshwater turtles, but can also parasitize frogs, salamanders and snakes (Li et al. 2014). Larval forms have been reported from a wide spectrum of hosts in various localities in Middle-America (e.g. Aguirre-Macedo et al. 2001; Sandlund et al. 2010).

The most widely distributed parasites among profundulids are the nematodes $R$. salgadoi and S. humbertoi, and the digenean P. blancoi sensu Salgado-Maldonado et al. (2011b), which are found in ten, eight and nine species of profundulids, in 38, 38 and 20 localities across Middle-America, respectively. Among these localities, prevalence and mean intensity values are quite variable (see Table 2). Prevalence varies between 2 and $100 \%$ for the different helminth species, although mean intensity values are usually very low (between one and five helminths per infected host), except for two 
Table 3. Host-parasite list. Key: $A=$ Adult, $M=$ Metacercariae, $L=$ Larvae.

\begin{tabular}{|c|c|c|}
\hline Host & Helminth parasite & Reference \\
\hline $\begin{array}{l}\text { Profundulus balsanus } \\
\text { Ahl, } 1935\end{array}$ & $\begin{array}{l}\text { Digenea } \\
\text { Ascocotyle (Ascocotyle) felippei (M) } \\
\text { Centrocestus formosanus (M) } \\
\text { Clinostomum sp. (M) } \\
\text { Diplostomidae gen. sp. (M) } \\
\text { Phyllodistomum spinopapillatum (A) } \\
\text { Posthodiplostomum minimum (M) } \\
\text { Paracreptotrema blancoi (A) } \\
\\
\text { Paracreptotrema profundulusi (A) } \\
\text { Saccocoelioides lamothei (A) } \\
\text { Monogenea } \\
\text { Gyrodactylus sp. (A) } \\
\text { Urocleidoides sp. (A) } \\
\text { Nematoda } \\
\text { Eustrongylides sp. (L) } \\
\text { Rhabdochona salgadoi (A) } \\
\text { Spinitectus humbertoi (A) }\end{array}$ & $\begin{array}{l}\text { Pinacho-Pinacho et al. (2014) } \\
\text { Pinacho-Pinacho et al. (2014) } \\
\text { Pinacho-Pinacho et al. (2014) } \\
\text { This study } \\
\text { Pinacho-Pinacho et al. (2014) } \\
\text { Pinacho-Pinacho et al. (2014) } \\
\text { Pérez-Ponce de León et al. (2015) } \\
\text { This study } \\
\text { Pinacho-Pinacho et al. (2014) } \\
\text { This study } \\
\text { Salgado-Maldonado et al. (2011b) } \\
\text { Pinacho-Pinacho et al. (2014) } \\
\text { Salgado-Maldonado et al. (2014) } \\
\text { This study } \\
\text { Salgado-Maldonado et al. (2011b) } \\
\text { Pinacho-Pinacho et al. (2014) } \\
\text { Salgado-Maldonado et al. (2014) } \\
\text { Pinacho-Pinacho et al. (2014) } \\
\text { This study } \\
\\
\text { Pinacho-Pinacho et al. (2014) } \\
\text { Pinacho-Pinacho et al. (2014) } \\
\\
\text { Pinacho-Pinacho et al. (2014) } \\
\text { Caspeta-Mandujano and Moravec (2000) } \\
\text { Pinacho-Pinacho et al. (2014) } \\
\text { Salgado-Maldonado et al. (2014) } \\
\text { Caspeta-Mandujano and Moravec (2000) } \\
\text { Pinacho-Pinacho et al. (2014) } \\
\text { Salgado-Maldonado et al. (2014) } \\
\text { This study }\end{array}$ \\
\hline $\begin{array}{l}\text { Profundulus } \\
\text { candalarius Hubbs, } \\
1924\end{array}$ & $\begin{array}{l}\text { Digenea } \\
\text { Clinostomum sp. (M) } \\
\text { Phyllodistomum inecoli (A) } \\
\text { Paracreptotrema blancoi (A) } \\
\text { Cestoda } \\
\text { Bothriocephalus acheilognathi (A) } \\
\\
\text { Nematoda } \\
\text { Eustrongylides sp. (L) } \\
\text { Spinitectus humbertoi (A) }\end{array}$ & $\begin{array}{l}\text { This study } \\
\text { This study } \\
\text { This study } \\
\text { Velazquez-Velazquez et al. (2015) } \\
\text { This study } \\
\text { This study } \\
\text { This study }\end{array}$ \\
\hline $\begin{array}{l}\text { Profundulus } \\
\text { guatemalensis } \\
\text { (Günther, 1866) }\end{array}$ & $\begin{array}{l}\text { Digenea } \\
\text { Paracreptotrema blancoi (A) } \\
\text { Monogenea } \\
\text { Urocleidoides sp. (A) } \\
\text { Cestoda } \\
\text { Bothriocephalus acheilognathi (A) } \\
\text { Nematoda } \\
\text { Rhabdochona salgadoi (A) } \\
\text { Spinitectus humbertoi (A) }\end{array}$ & $\begin{array}{l}\text { Salgado-Maldonado et al. (2014) } \\
\text { This study } \\
\text { This study } \\
\text { This study } \\
\text { Salgado-Maldonado et al. (2014) } \\
\text { This study } \\
\text { Salgado-Maldonado et al. (2014) } \\
\text { This study }\end{array}$ \\
\hline
\end{tabular}




\begin{tabular}{|c|c|c|}
\hline Host & Helminth parasite & Reference \\
\hline $\begin{array}{l}\text { Profundulus } \\
\text { hildebrandi Miller, } \\
1950\end{array}$ & $\begin{array}{l}\text { Cestoda } \\
\quad \text { Bothriocephalus acheilognathi (A) }\end{array}$ & $\begin{array}{l}\text { Velázquez-Velázquez et al. (2011) } \\
\text { This study }\end{array}$ \\
\hline $\begin{array}{l}\text { Profundulus kreiseri } \\
\text { Matamoros, Schaefer, } \\
\text { Hernández \& } \\
\text { Chakrabarty, } 2012\end{array}$ & $\begin{array}{l}\text { Digenea } \\
\quad \text { Paracreptotrema blancoi (A) } \\
\text { Nematoda } \\
\quad \text { Rhabdochona salgadoi (A) } \\
\text { Spinitectus humbertoi (A) }\end{array}$ & $\begin{array}{l}\text { Salgado-Maldonado et al. (2014) } \\
\text { This study } \\
\text { Salgado-Maldonado et al. (2014) } \\
\text { This study } \\
\text { Salgado-Maldonado et al. (2014) }\end{array}$ \\
\hline $\begin{array}{l}\text { Profundulus labialis } \\
\text { (Günther, 1866) }\end{array}$ & $\begin{array}{l}\text { Digenea } \\
\text { Paracreptotrema blancoi (A) } \\
\text { Nematoda } \\
\text { Rhabdochona salgadoi (A) } \\
\text { Spinitectus humbertoi (A) } \\
\text { Spinitectus mariaisabelae (A) }\end{array}$ & $\begin{array}{l}\text { This study } \\
\text { Salgado-Maldonado et al. (2011a) } \\
\text { Salgado-Maldonado et al. (2014) } \\
\text { Salgado-Maldonado et al. (2014) } \\
\text { Salgado-Maldonado et al. (2011a) }\end{array}$ \\
\hline $\begin{array}{l}\text { Profundulus oaxacae } \\
\text { (Meek, 1902) }\end{array}$ & $\begin{array}{l}\text { Digenea } \\
\text { Paracreptotrema blancoi (A) } \\
\text { Paracreptotrema profundulusi (A) } \\
\text { Nematoda } \\
\quad \text { Rhabdochona salgadoi (A) }\end{array}$ & $\begin{array}{l}\text { Salgado-Maldonado et al. (2014) } \\
\text { Salgado-Maldonado et al. (2011b) } \\
\text { Salgado-Maldonado et al. (2014) } \\
\text { Salgado-Maldonado et al. (2014) }\end{array}$ \\
\hline $\begin{array}{l}\text { Profundulus } \\
\text { portillorum } \\
\text { Matamoros \& } \\
\text { Schaefer, } 2010\end{array}$ & $\begin{array}{l}\text { Digenea } \\
\text { Paracreptotrema blancoi (A) } \\
\text { Allocreadiidae gen. sp. (A) } \\
\text { Cestoda } \\
\text { Bothriocephalus acheilognathi (A) } \\
\text { Nematoda } \\
\text { Rhabdochona salgadoi (A) } \\
\text { Spinitectus humbertoi (A) } \\
\text { Spiroxys sp. (L) }\end{array}$ & $\begin{array}{l}\text { This study } \\
\text { This study } \\
\text { Salgado-Maldonado et al. (2015) } \\
\text { Salgado-Maldonado et al. (2014) } \\
\text { This study } \\
\text { This study }\end{array}$ \\
\hline $\begin{array}{l}\text { Profundulus punctatus } \\
\text { (Günther, 1866) }\end{array}$ & $\begin{array}{l}\text { Digenea } \\
\text { Ascocotyle (Ascocotyle) felippei (M) } \\
\text { Centrocestus formosanus (M) } \\
\text { Clinostomum sp. (M) } \\
\text { Phyllodistomum inecoli (A) } \\
\text { Paracreptotrema blancoi (A) } \\
\text { Paracreptotrema profundulusi (A) } \\
\text { Monogenea } \\
\text { Urocleidoides sp. (A) } \\
\text { Nematoda } \\
\text { Contracaecum sp. (L) } \\
\text { Eustrongylides sp. (L) } \\
\text { Rhabdochona salgadoi (A) } \\
\text { Spinitectus humbertoi (A) } \\
\text { Spinitectus mariaisabelae (A) }\end{array}$ & $\begin{array}{l}\text { This study } \\
\text { This study } \\
\text { Salgado-Maldonado et al. (2011a) } \\
\text { This study } \\
\text { Pérez-Ponce de León et al. (2015) } \\
\text { Salgado-Maldonado et al. (2011b) } \\
\text { Salgado-Maldonado et al. (2014) } \\
\text { This study } \\
\text { Salgado-Maldonado et al. (2011b) } \\
\text { Salgado-Maldonado et al. (2014) } \\
\text { This study } \\
\text { Salgado-Maldonado et al. (2011a) } \\
\text { This study } \\
\text { Salgado-Maldonado et al. (2011a) } \\
\text { Salgado-Maldonado et al. (2014) } \\
\text { Salgado-Maldonado et al. (2011a) } \\
\text { This study } \\
\text { Salgado-Maldonado et al. (2014) } \\
\text { Caspeta-Mandujano et al. (2007) } \\
\text { Salgado-Maldonado et al. (2011a) }\end{array}$ \\
\hline
\end{tabular}




\begin{tabular}{l|l|l}
\hline Host & Helminth parasite & Reference \\
\hline Profundulus sp. 1 & $\begin{array}{l}\text { Nematoda } \\
\text { Rhabdochona salgadoi (A) } \\
\text { Spinitectus humbertoi (A) }\end{array}$ & $\begin{array}{l}\text { Salgado-Maldonado et al. (2014) } \\
\text { Salgado-Maldonado et al. (2014) }\end{array}$ \\
\hline $\begin{array}{l}\text { Profundulus sp. 2 } \\
\text { Phyllodistomum inecoli (A) } \\
\text { Paracreptotrema blancoi (A) }\end{array}$ & $\begin{array}{l}\text { Pérez-Ponce de León et al. (2015) } \\
\text { Salgado-Maldonado et al. (2014) } \\
\text { Salgado-Maldonado et al. (2011b) } \\
\text { This study } \\
\text { Salgado-Maldonado et al. (2014) } \\
\text { Salgado-Maldonado et al. (2011b) } \\
\text { This study }\end{array}$ \\
& $\begin{array}{l}\text { Paracrototrema profundulusi (A) } \\
\text { Shatoda } \\
\text { Rhabdochona salgadoi (A) }\end{array}$ & $\begin{array}{l}\text { Salgado-Maldonado et al. (2014) } \\
\text { Salgado-Maldonado et al. (2011a) }\end{array}$ \\
\hline
\end{tabular}

larval forms, the heterophyids C. formosanus and Ascocotyle (Ascocotyle) felippei Travassos, 1928. These two species reached mean intensity levels as high as 821.6 and 165.4 larvae per infected host, respectively. Among adults, the nematodes $S$. humbertoi and $R$. salgadoi reached mean intensity values usually higher than five worms per infected host among the various localities.

In terms of the species richness of the helminths in relation to the host species, Profundulus balsanus is the species with the highest diversity, since it is parasitized by 14 species, followed by $P$. punctatus with 12 , and $P$. candalarius and $P$. portillorum with six (Table 3). Finally, $P$. oaxacae, $P$. kreiseri and $P$. hildebrandi possess a depauperate fauna, with only three, three and one species, respectively.

\section{Discussion}

Fish were collected at 26 localities in southern Mexico, Guatemala and Honduras, and a total of 267 individual fish belonging to nine species of Profundulus was examined for helminth parasites. The inventory was completed by adding these records to the previous parasite surveys conducted on members of this fish group endemic to Middle-America. Interestingly, the number of individual hosts studied for helminths of this fish group has increased significantly during a two-year period across the entire distributional range, and it seems that only two new species were found. Firstly, a detailed morphological evaluation of the specimens recorded herein as Urocleidoides sp. indicate they represent an undescribed species, which will be formally described in a separate paper. A thorough revision of the morphology of the specimens identified as the trematode Paracreptotrema blancoi by Salgado-Maldonado et al. (2011b), along with the new samples obtained in this study, allowed us to determine that they in fact represent not only a new species but a new genus. The new species is readily distinguished by the size of the ventral sucker and by having a more restricted vitellarium, a shorter cirrus sac and caeca that bifurcate at the level of the ventral sucker and end at the level of the testes. Since information was also gathered from sequences 


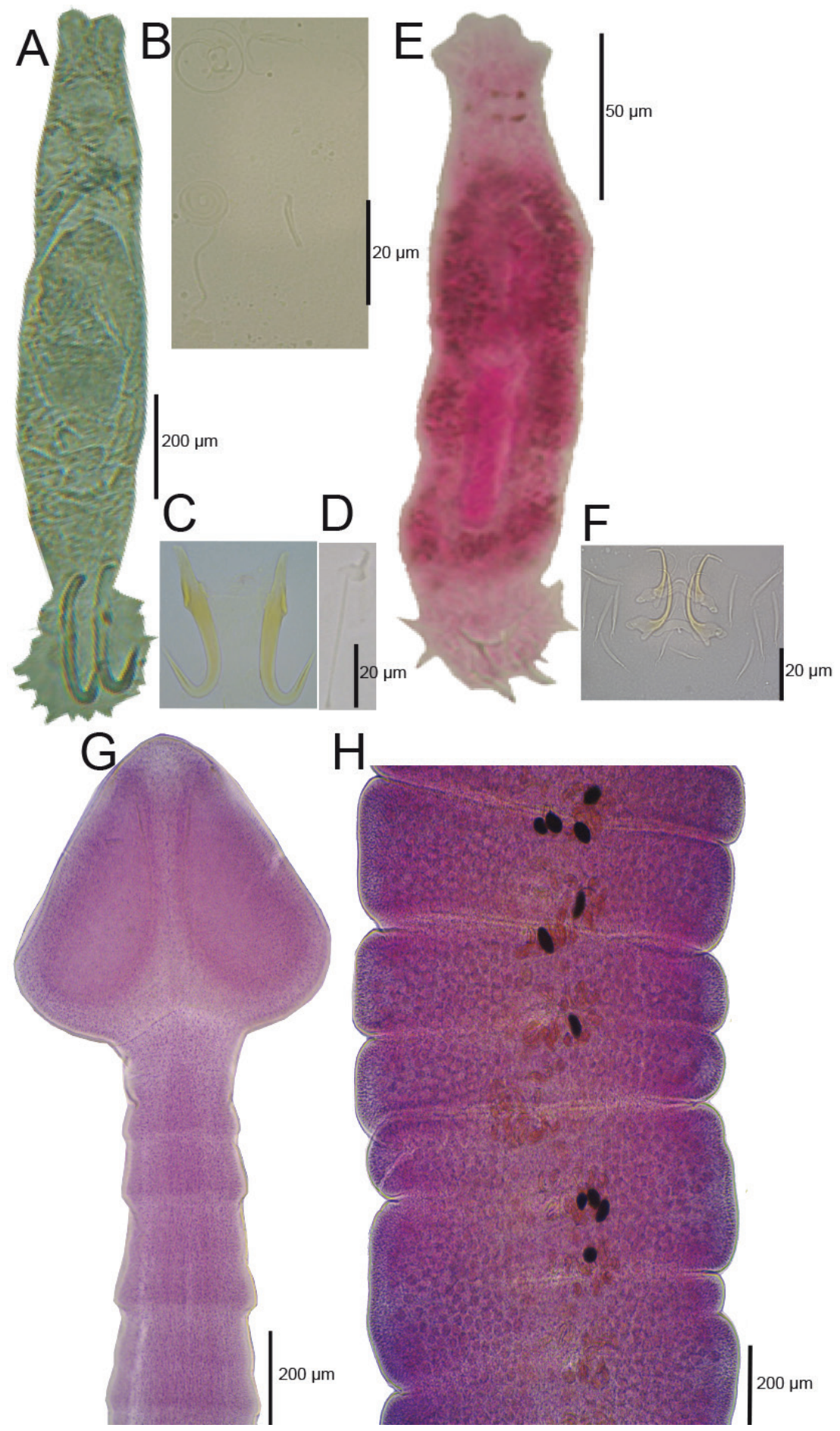

Figure 3. Species of monogeneans and the single cestode found in Profundulus spp. A-D Gyrodactylus sp. E-F Urocleidoides sp. G-H Bothriocephalus acheilognathi. 


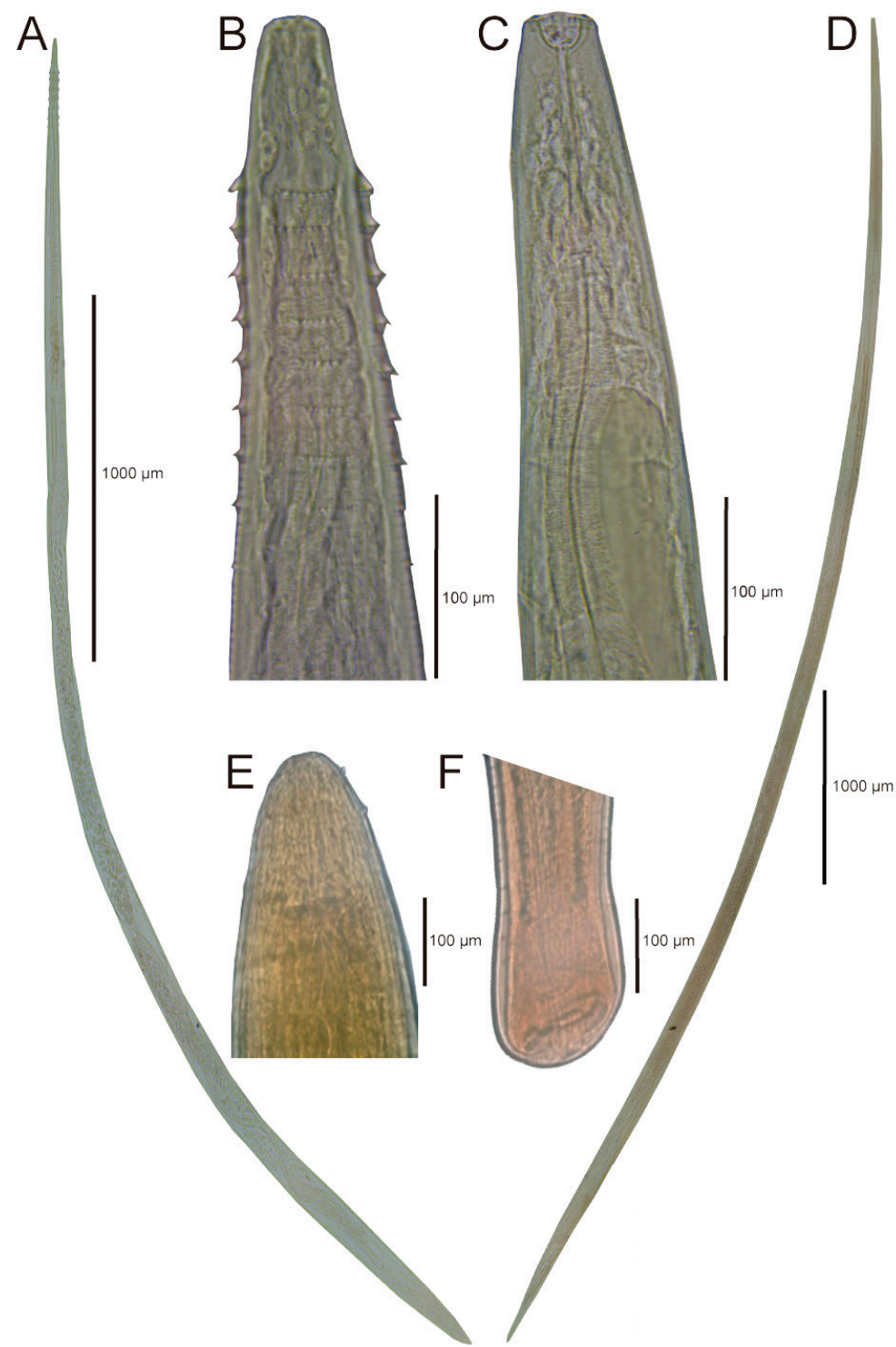

Figure 4. Species of nematodes found in Profundulus. A-B Spinitectus humbertoi C-D Rhabdochona salgadoi $\mathbf{E}-\mathbf{F}$ Eustrongylides sp.

of the 28S rRNA gene and scanning electron microscopy micrographs, the new species will be formally described in a separate paper. The record in this checklist is presented provisionally, under the original designation of the species, as P. blancoi sensu SalgadoMaldonado et al. (2011b).

Six adult helminth species are considered to be part of the biogeographical 'core' helminth fauna of profundulids. As discussed by Pérez-Ponce de León and Choudhury (2002), for a parasite taxon to be considered part of a biogeographical core, it 
must not only be widely distributed but must also be characteristically associated with and restricted to a monophyletic group of host species (see also Choudhury and Dick 1998), even if it is not present in all host species of that group. This concept was actually used to describe the pattern of host-specificity among the helminth parasite fauna of freshwater fishes in Mexico (Pérez-Ponce de León and Choudhury 2005); this was based on the premise that particular host-groups are characteristically associated with a biogeographical 'core' helminth fauna and that such host specificity strongly influences their biogeography. These authors tested three predictions based on that fundamental hypothesis of 'core' parasite faunas: (1) that the parasite fauna is largely circumscribed by higher levels of monophyletic host taxa (families, orders, etc.), and that this pattern is independent of areas; (2) that areas within a certain biogeographical region, and consequently with a similar fish composition, will have more similar parasite faunas compared with areas with a less similar fish faunal composition; and (3) that 'core' parasite faunas persist to some extent in transitional areas (areas where Nearctic and Neotropical species are sympatric) with limited host-sharing. The current results on the helminth fauna of Profundulus spp. along its distributional range in Middle-America further corroborate the three predictions.

This represents the second complete inventory of a freshwater fish group. Martínez-Aquino et al. (2014) recently published the inventory of the helminth parasites of goodeines, an endemic subfamily from central and a few areas of northern Mexico. Both groups belong in the order Cyprinodontiformes, and molecular phylogenetic analyses show that they are sister taxa (Webb et al. 2004, Doadrio and Domínguez 2004). Based on the premise that comprehensive data on the inventory of a particular host group is fundamental to a better understanding of the historical biogeography and evolutionary history of host-parasite associations, the information presented in this paper, along with the one for the goodeines, will allow us to discuss factors that have shaped the biogeographical and diversification patterns of parasites and hosts within a phylogenetic framework, and, to the best of our knowledge, this is the first time that these types of data have been produced.

There are, however, some notable differences between the biogeography of the Goodeinae and Profundulidae. The Goodeinae is an endemic fish component of central and northern Mexico which experienced an important adaptive radiation and contains 45 species (Domínguez-Domínguez et al. 2010). The helminth fauna of extant species (some of them have gone extinct recently due to habitat degradation) includes 51 species, according to the examination of almost 8,300 individual fish representing 36 species allocated to 18 genera, studied in 113 localities across central and northwestern Mexico (Martínez-Aquino et al. 2014). In contrast, Profundulus possesses only 11 species and is the only genus within the Profundulidae. These fish did not experience the same level of diversification as goodeines, and their distributional range comprises an area of Middle-America from central Mexico southwards to Honduras. Apparently, the Balsas depression establishes the distributional limit for both fish groups, since goodeines have the southernmost distribution range in the Balsas drainage, whereas profundulids reach their northernmost distribution in the same basin. However, these 
fish families do not occur sympatrically at any location. Since the Balsas River basin is the result of a geological event known as the Balsas Portal, which represents a marine transgression that occurred during the Mid-Cretaceous period (see DomínguezDomínguez and Pérez-Ponce de León 2009, and references therein), it cannot be ruled out that this was the geological event that caused the divergence between goodeines and profundulids from a common ancestor, despite a molecular clock analysis showing that the ancestral split occurred during the Mid-Miocene, approximately 16 million years ago (see Doadrio and Domínguez 2004).

Irrespective of the biogeographical history of the ancestor of both profundulids and goodeids, and the subsequent radiation of the latter, the former did not diversify in the same way as goodeids did. Adaptive radiation of goodeines in central Mexico, following a complex geological and hydrological history (see Domínguez-Domínguez et al. 2010), resulted in a higher species richness, and this may have influenced their parasite fauna, contrasting the 51 helminth species that parasitize goodeines with only 20 species in profundulids. Interestingly, the helminth species composition in both host groups is relatively similar. The core helminth parasite fauna includes members of the Allocreadiidae Looss, 1902, Gorgoderidae Looss, 1901 and Haploporidae Nicoll, 1914 among the digeneans, members of the monogenean genus Gyrodactylus von Nordmann, 1832 and members of the nematode genus Rhabdochona Railliet, 1916. For instance, while goodeines are infected by two species of the allocreadiid genus Margotrema Lamothe-Argumedo, 1970, profundulids are infected by two species of the allocreadiid genus Paracreptotrema Choudhury, Pérez-Ponce de León, Brooks \& Daverdin, 2006. Both host groups are parasitized by two species of Phyllodistomum Braun, 1899 , and by two species of Rhabdochona. Likewise, scarce phylogenetic information is available to make strong comparisons, and a robust pattern cannot be established in the absence of a phylogenetic framework. However, the few available data show that the presence of congeners of different helminth groups in goodeines and profundulids is not the result of a historical association but of colonization (Brooks and McLennan 1993). In the morphological phylogenetic analysis of species of Rhabdochona by Mejía-Madrid et al. (2007), R. lichtenfelsi Sánchez-Alvarez, García-Prieto \& PérezPonce de León, 1998 (a common and widely distributed parasite of goodeines) and $R$. salgadoi (a common and widely distributed species in Profundulus) are not close relatives, although, needless to say, the phylogenetic analysis was not fully resolved and the morphology-based phylogeny may not be robust. In contrast, in the case of the allocreadiids, recently published molecular phylogenetic analyses clearly indicate that Margotrema spp. (in goodeines) and Paracreptotrema (in profundulids) are not sister taxa, since Margotrema clusters with species usually found in Nearctic fishes, whereas Paracreptotrema is the sister taxon to other allocreadiids that parasitize characids (RazoMendivil et al. 2014, a group of fish with a Neotropical origin, precluding any speculation about the speciation event that may have caused their diversification either in goodeines or profundulids).

As suggested by Pérez-Ponce de León and Choudhury (2010), molecular data are fundamental to better understanding patterns of diversity among the freshwater fish 
parasite fauna, but also to establishing sister group relationships among newly discovered species with respect to those already described. At present, it seems plausible to propose that the helminth fauna of goodeines was secondarily acquired from Nearctic fishes, whereas profundulids obtained their helminths from other Neotropical freshwater fishes, i.e. their parasites are the result of host-switching events following colonization from other, most probably unrelated, hosts. But this needs to be determined by proper molecular co-phylogenetic analyses. The data generated thus far will enable us to conduct such analyses in the near future and to contribute to a better understanding of the evolution and biogeography of the freshwater fish helminth parasite fauna.

\section{Acknowledgments}

We are grateful to Carla Mena, Eufemia Arenas, and Marly Martínez for their help during field work. We thank B. Mendoza-Garfías for processing some of the specimens, and L. García-Prieto for providing literature and specimens for morphological comparison deposited at the CNHE. This research was supported by grants from the Programa de Apoyo a Proyectos de Investigación e Inovación Tecnológica (PAPIITUNAM) IN207213 and IN219904 to MGV and GPPL, respectively, and the Consejo Nacional de Ciencia y Tecnología (CONACYT) 179048 to MGV. C.D.P.P. thanks the support of the Programa de Posgrado en Ciencias Biológicas, UNAM and CONACYT for granting a scholarship to complete his PhD program. JSHO and C.A.M. benefited from a Postdoctoral Fellowship of the Dirección General Asuntos del Personal Académico (DGAPA-UNAM).

\section{References}

Aguirre-Macedo ML, Scholz T, González-Solís D, Vidal-Martínez VM, Posel P, Arjona-Torres G, Siu-Estrada E, Dumailo S (2001) Larval helminths parasitizing freshwater fishes from the Atlantic coast of Nicaragua. Comparative Parasitology 68: 42-51.

Andrade-Gómez L (2015) Diferenciación morfológica y molecular de cuatro especies de tremátodos de la familia Haploporidae Nicoll, 1914 (Digenea) parásitos de peces dulceacuícolas de México y partes de América Central. Bachelor Thesis, Universidad Nacional Autónoma de México, Mexico, DF, 101 pp.

Brooks RD, McLennan DA (1993) Parascript: parasites and the language of evolution. Smithsonian Institution. Press, Washington DC, $430 \mathrm{pp}$.

Bush AO, Lafferty KD, Lotz JM, Shostak AW (1997) Parasitology meets ecology on its own terms: Margolis et al. revisited. Journal of Parasitology 83: 575-583. doi: 10.2307/3284227

Caspeta-Mandujano JM, Cabańas-Carranza G, Salgado-Maldonado G (2007) Spinitectus mariaisabelae n. sp. (Nematoda: Cystidicolidae) from the intestine of the freshwater fish Profundulus punctatus (Cyprinodontiformes) in Mexico. Helminthologia 44: 103-106. doi: 10.2478/s11687-007-0014-8 
Caspeta-Mandujano JM, Moravec F (2000) Two new intestinal nematodes of Profundulus labialis (Pisces, Cyprinodontidae) from freshwaters in Mexico. Acta Parasitologica 45: 332-339. Choudhury A, Dick TA (1998) Patterns and determinants of helminth communities in the Acipenseridae (Actinopterygii: Chondrostei), with special reference to the lake sturgeon, Acipenser fulvescens. Canadian Journal of Zoology 76: 330-349. doi: 10.1139/cjz-76-2-330 Choudhury A, Zheng S, Pérez-Ponce de León G, Martínez-Aquino A, Brosseau C, Gale E (2013) The invasive Asian fish tapeworm, Bothriocephalus acheilognathi Yamaguti, 1934, in the Chagres River/Panama Canal drainage, Panama. BioInvasions Records 2: 99-104. doi: 10.3391/bir.2013.2.2.02

Doadrio I, Domínguez-Domínguez O (2004) Phylogenetic relationships within the fish family Goodeidae based on cytochrome b sequence data. Molecular Phylogenetics and Evolution 31: 416-430. doi: 10.1016/j.ympev.2003.08.022

Doadrio I, Carmona JA, Martínez E, de Sostoa A (1999) Genetic variation and taxonomic analysis of the subgenus Profundulus. Journal of Fish Biology 55: 751-766. doi: 10.1111/ j.1095-8649.1999.tb00715.x

Domínguez-Domínguez O, Pedraza-Lara C, Gurrola-Sánchez N, Perea S, Pérez-Rodríguez R, Israde-Alcántara I, Garduño-Monroy VH, Doadrio I, Pérez-Ponce de León G, Brooks DR (2010) Historical biogeography of the Goodeinae (Cyprinodontiforms). In: Uribe-Aranzabal MC, Grier H (Eds) Viviparous fishes II. New Life Publications, Homestead, 33-74.

Domínguez-Domínguez O, Pérez-Ponce de León G (2009) ¿La mesa central de México es una provincia biogeográfica? Análisis descriptivo basado en componentes bióticos dulceacuícolas. Revista Mexicana de Biodiversidad 80: 835-852.

Ergens R (1969) The suitability of ammonium picrate-glycerin in preparing slides of lower Monogenoidea. Folia Parasitologica 16: 320.

González-Díaz AA, Díaz-Pardo E, Soria-Barreto M, Rodiles-Hernández R (2005) Análisis morfométrico de los peces del grupo labialis, género Profundulus (Cyprinodontiformes: Profundulidae), en Chiapas, México. Revista Mexicana de Biodiversidad 76: 55-61.

Li L, Hasegawa H, Roca V, Xu Z, Guo YN, Sato A, Zhang LP (2014) Morphology, ultrastructure and molecular characterisation of Spiroxys japonica Morishita, 1926 (Spirurida: Gnathostomatidae) from Pelophylax nigromaculatus (Hallowell) (Amphibia: Ranidae). Parasitology Research 113: 893-901. doi: 10.1007/s00436-013-3720-9

Luque JL, Poulin R (2007) Metazoan parasite species richness in Neotropical fishes: hotspots and the geography of biodiversity. Parasitology 134: 865-878. doi: 10.1017/ S0031182007002272

Martínez-Aquino A, Mendoza-Palmero CA, Aguilar-Aguilar R, Pérez-Ponce de León G (2014) Checklist of helminth parasites of Goodeinae (Osteicthyes: Cyprinodontiformes: Goodeidae), an endemic subfamily of freshwater fishes from Mexico. Zootaxa 3856: 151-191. doi: 10.11646/zootaxa.3856.2.1

Martínez-Ramírez E, Doadrio-Villarejo I, de Sostoa-Fernández A (2004) Peces Continentales. In: García-Mendoza AJ, Ordóñez MJ, Briones-Salas M (Eds) Biodiversidad de Oaxaca. Instituto de Biología, Universidad Nacional Autónoma de México, Fondo Oaxaqueño para la Conservación de la Naturaleza and World Wildlife Fund, México, DF, 357-373. 
Matamoros WA, Schaefer JF (2010) A new species of Profundulus (Cyprinodontiformes: Profundulidae) from the Honduran central highlands. Journal of Fish Biology 76: 1498-1507. doi: 10.1111/j.1095-8649.2010.02596.x

Matamoros WA, Schaefer JF, Hernández CL, Chakrabarty P (2012) Profundulus kreiseri, a new species of Profundulidae (Teleostei, Cyprinodontiformes) from Northwestern Honduras. Zookeys 227: 49-62. doi: 10.3897/zookeys.227.3151

Mejía-Madrid H, Choudhury A, Pérez-Ponce de León G (2007) Phylogeny and biogeography of Rhabdochona Railliet, 1916 (Nematoda: Rhabdochonidae) species from the Americas. Systematic Parasitology 67: 1-18. doi: 10.1007/s11230-006-9065-3

Miller RR (1955) A systematic review of the middle American fishes of the genus Profundulus. Miscellaneous Publications Museum of Zoology University of Michigan, Ann Arbor, Michigan 92: 1-64.

Miller RR, Minckley WL, Norris SM (2005) Freshwater fishes of Mexico. The University of Chicago Press, Chicago, 490 pp.

Pérez-Ponce de León G, Choudhury A (2002) Adult Endohelminth Parasites of Ictalurid Fishes (Osteichthyes: Ictaluridae) in Mexico: Empirical Evidence for Biogeographical Patterns. Comparative Parasitology 69: 10-19. doi: 10.1654/1525-2647(2002)069[0010:AEPOIF]2.0.CO;2

Pérez-Ponce de León G, Choudhury A (2005) Biogeography of helminth parasites of freshwater fishes in Mexico: the research for patterns and processes. Journal of Biogeography 32: 645-649. doi: 10.1111/j.1365-2699.2005.01218.x

Pérez-Ponce de León G, Choudhury A (2010) Parasite inventories and DNA-based taxonomy: lessons from helminths of freshwater fishes in a megadiverse country. Journal of Parasitology 96: 236-244. doi: 10.1645/GE-2239.1

Pérez-Ponce de León G, Pinacho-Pinacho CD, Mendoza-Garfias B, García-Varela M (2015) Phyllodistomum spinopapillatum sp. nov. (Digenea: Gorgoderidae), from the Oaxaca killifish Profundulus balsanus (Osteichthyes: Profundulidae) in Mexico, with new host and locality records of $P$. inecoli: Morphology, ultrastructure and molecular evidence. Acta Parasitologica 60: 298-307. doi: 10.1515/ap-2015-0042

Pinacho-Pinacho CD, Pérez-Ruiz MÁ, Sereno-Uribe AL, García-Varela M, Martínez-Ramírez E (2014) Richness and similarity of helminth communities of the freshwater fish Profundulus punctatus (Pisces: Cyprinodontidae) from Oaxaca, Mexico. Revista Mexicana de Biodiversidad 85: 1129-1138. doi: 10.7550/rmb.41776

Razo-Mendivil U, Pérez-Ponce de León G, Rubio-Godoy M (2014) Testing the Systematic Position and Relationships of Paracreptotrema heterandriae Within the Allocreadiidae Through Partial 28s rRNA Gene Sequences. Journal of Parasitology 10: 37-541. doi: $10.1645 / 13-421.1$

Salgado-Maldonado G, Caspeta-Mandujano JM, Moravec F, Soto-Galera E, Rodiles-Hernández R, Cabañas-Carranza G, Montoya-Mendoza J (2011a) Helminth parasites of freshwater fish in Chiapas, Mexico. Parasitology Research 108: 31-59. doi: 10.1007/s00436-010-2035-3

Salgado-Maldonado G, Caspeta-Mandujano JM, Martínez-Ramírez E (2011b) Paracreptotrema profundulusi n. sp. and P. blancoi Choudhury, Pérez Ponce de León, Brooks, and Daverdin, 2006 (Trematoda: Allocreadiidae) from freshwater fishes of the genus Profundulus 
(Teleostei: Profundulidae) in Southern Mexico. Journal of Parasitology 97: 707-712. doi: 10.1645/GE-2662.1

Salgado-Maldonado G, Matamoros WA, Caspeta-Mandujano JM, Martínez-Ramírez E, Mendoza-Franco E, Velázquez-Velázquez E (2014) Range extension of helminth parasites of Profundulus spp. (Teleostei: Profundulidae) from southern Mexico and Central America. Check List 10: 1507-1513. doi: 10.15560/10.6.1507

Salgado-Maldonado G, Matamoros WA, Kreiser BR, Caspeta-Mandujano JM, MendozaFranco EF (2015) First record of the invasive Asian fish tapeworm Bothriocephalus acheilognathi in Honduras, Central America. Parasite 22: 1-5. doi: 10.1051/parasite/2015007 Sandlund OT, Daverdin RH, Choudhury A, Brooks DR, Diserud OH (2010) A survey of freshwater fishes and their macroparasites in the Guanacaste Conservation Area (ACG), Costa Rica. Norwegian Institute for Nature Research (NINA) Report 635, 45 pp.

Scholz T, Salgado-Maldonado G (2000) The introduction and dispersal of Centrocestus formosanus (Nishigori, 1924) (Digenea:Heterophyidae) in Mexico: A review. American Midland Naturalist 143: 185-200.

Sereno-Uribe AL, Pinacho-Pinacho CD, García-Varela M, Pérez-Ponce de León G (2013) Using mitochondrial and ribosomal DNA sequences to test the taxonomic validity of Clinostomum complanatum Rudolphi, 1814 in fish-eating birds and freshwater fishes in Mexico, with the description of a new species. Parasitology Research 112: 2855-2870. doi: 10.1007/s00436-013-3457-5

Velázquez-Velázquez E, Mendez-Gómez B, Salgado-Maldonado G, Matamoros W (2015) The invasive tapeworm Bothriocephalus acheilognathi Yamaguti, 1934 in the endangered killifish Profundulus candalarius Hubbs, 1924 in Chiapas, Mexico. BioInvasions Records 4. [in press] Velázquez-Velázquez E, González-Solís D, Salgado-Maldonado G (2011) Bothriocephalus acheilognathi (Cestoda) in the endangered fish Profundulus hildebrandi (Cyprinodontiformes), Mexico. Revista de Biología Tropical 59: 1099-1104. doi: 10.15517/rbt.v0i0.3382

Vidal-Martínez VM, Aguirre-Macedo ML, Scholz T, González-Solís D, Mendoza Franco EF (2001) Atlas of the helminth parasites of cichlid fish of Mexico. Academia, Praha, 165 pp. Webb SA, Graves JA, Macias-Garcia C, Magurran AE, Foighil DO, Ritchie MG (2004) Molecular phylogeny of the livebearing Goodeidae (Cyprinodontiformes). Molecular Phylogenetics and Evolution 30: 527-544. doi: 10.1016/S1055-7903(03)00257-4 